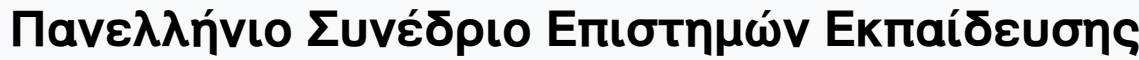

Tóp. 2016, Ap. 1

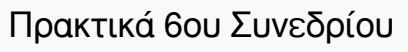

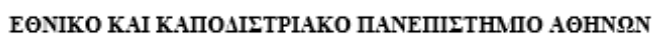

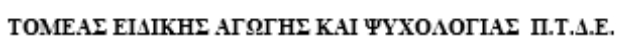

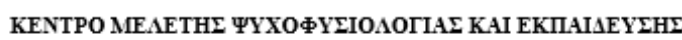

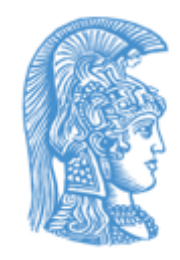

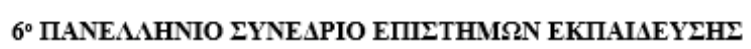

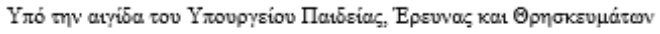

24-26 Iouviov 2016

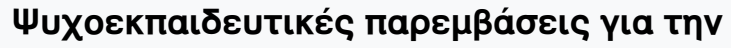

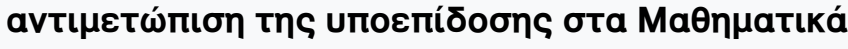

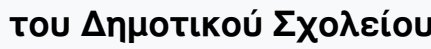

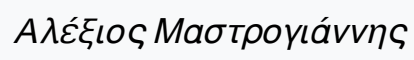

doi: $10.12681 /$ edusc.980

\section{ПIPAKTIKA IYNEAPIOY}

ISSN: 2529-1157

EIIMEAEIA:

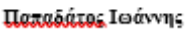

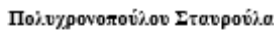

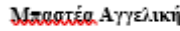

IOYNIOE 2016

\section{Bıß入ıорачıкń avaчopá:}

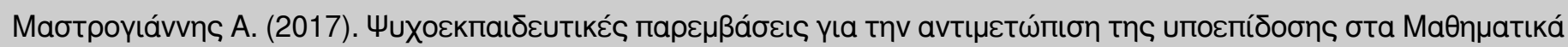

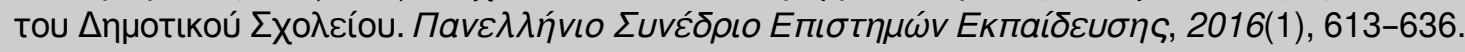

https://doi.org/10.12681/edusc.980 


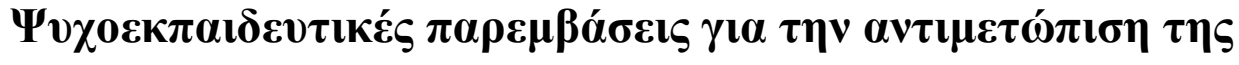

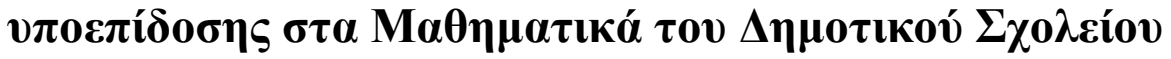

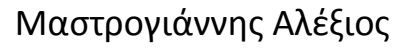

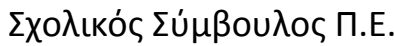

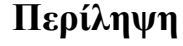

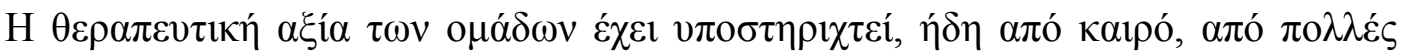

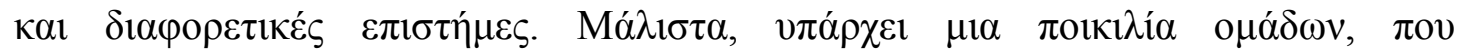

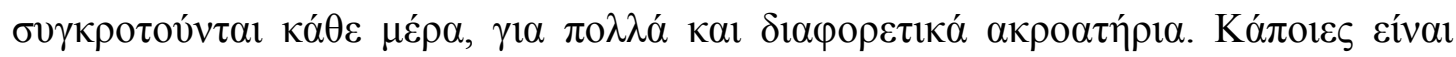

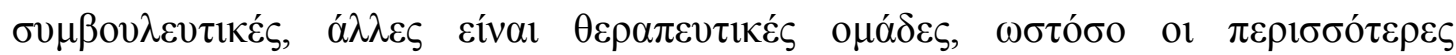

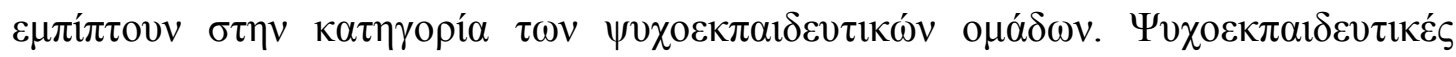

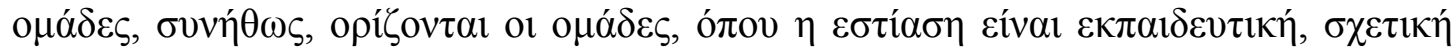

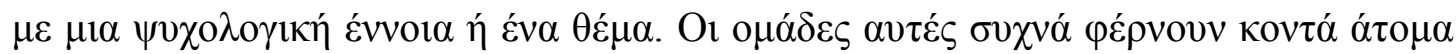

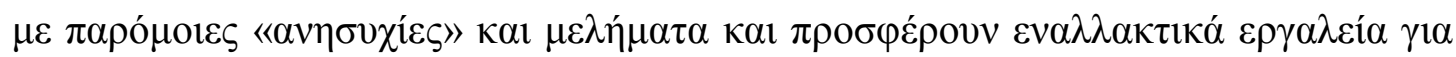

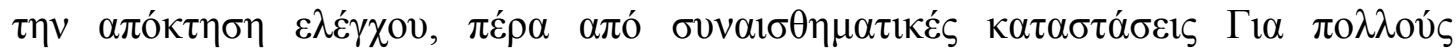

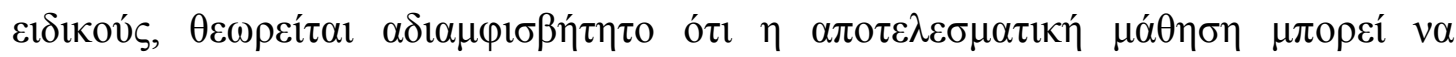

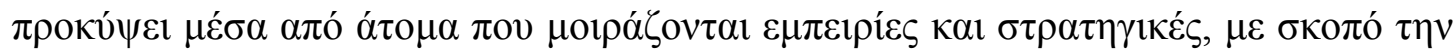

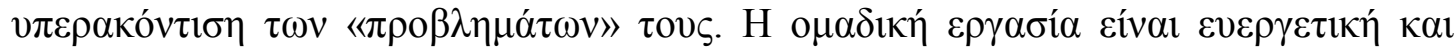

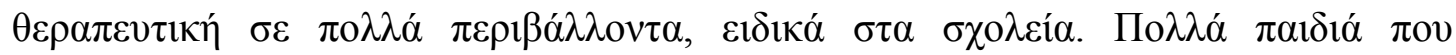

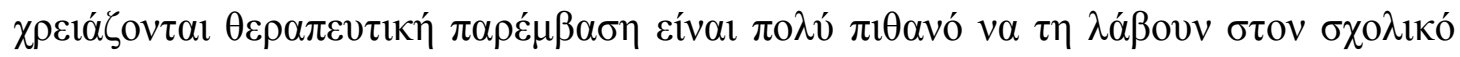

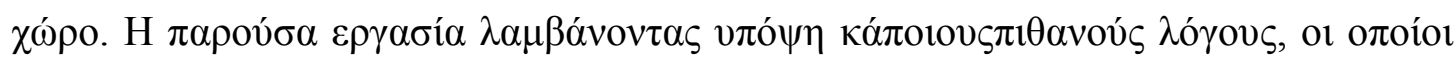

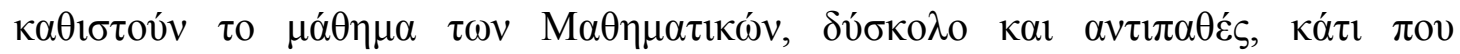

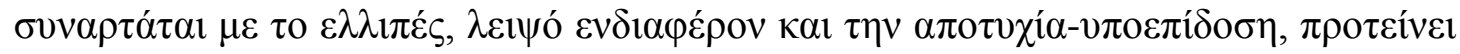

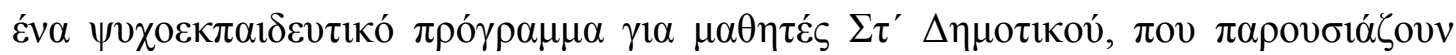

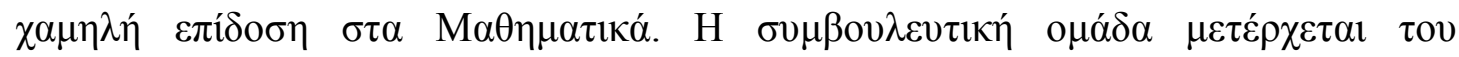

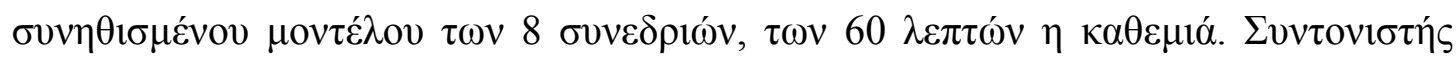

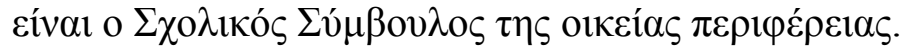

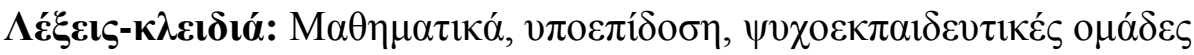

\begin{abstract}
The therapeutic benefits of group dynamics are nowadays confirmed by many fields of science. In fact, there is a wide range of groups which are created on a daily basis, for many different audiences. Some of them are advisory groups, others are therapeutic groups, but most of them fall into the category of Psychoeducational groups. Psychoeducational groups focus on educational matters, related to a psychological concept or theme. These groups often bring together people with similar concerns and offer alternatives on maintaining self-control over emotional situations. Many experts believe that effective learning can occur when people share experiences and strategies in order to deal with their personal problems. Teamwork is
\end{abstract}


beneficial and healing for many environments, especially in schools. For many children, who require a therapeutic intervention, is more likely to take it in school. Considering some possible reasons that make Maths a difficult and unpopular school subject, as much as the general lack of interest and the underachievement among schoolchildren, the present work suggests a psychoeducational program for students of 6th grade, who perform poorly in Maths. This intervention is carried out in 8 sessions, utilizing the usual model of 60 minutes each. The School Advisor of this region is the Coordinator of the group.

Keywords: Mathematics, underachievement, psychoeducational groups

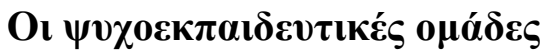

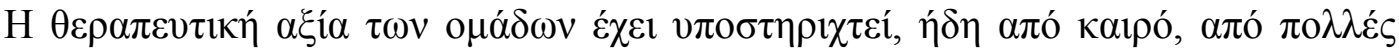

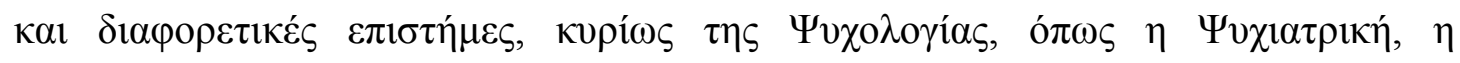

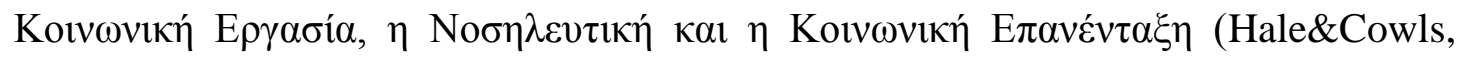

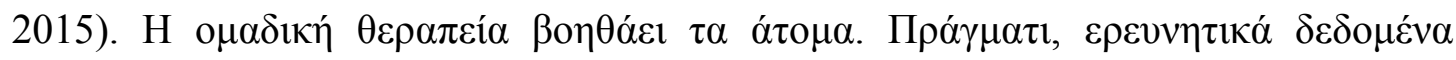

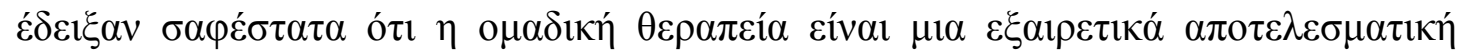

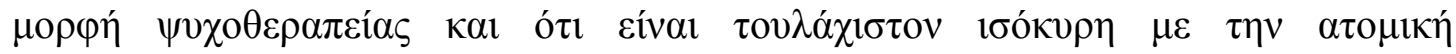

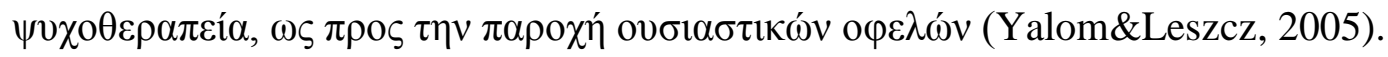

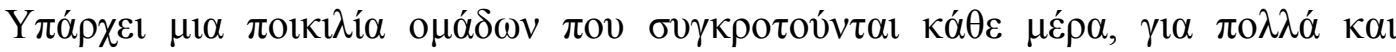

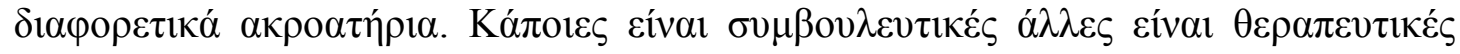

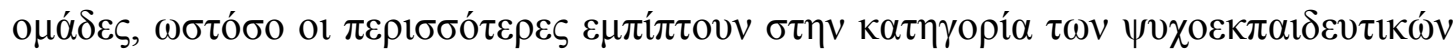

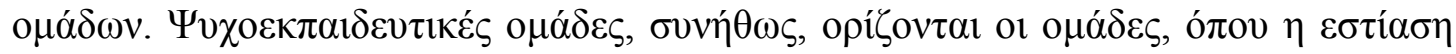

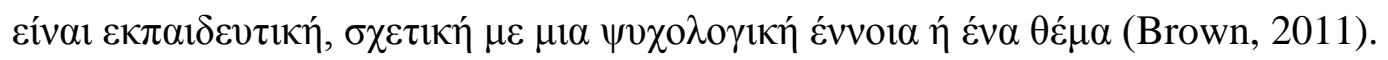

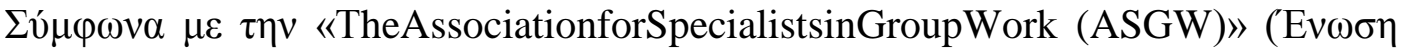

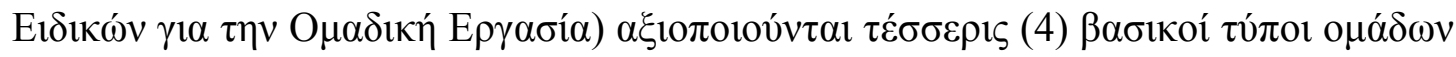

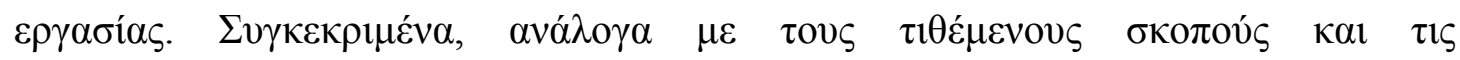

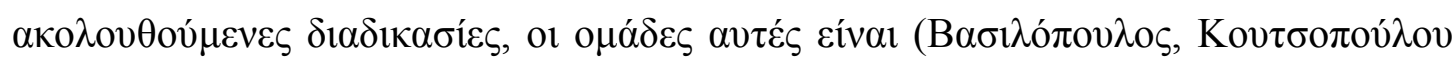

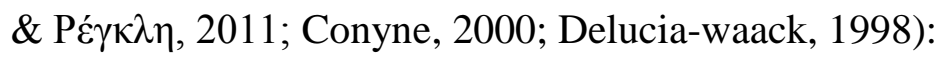

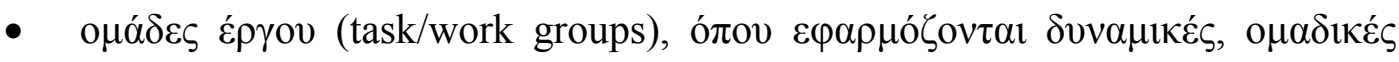

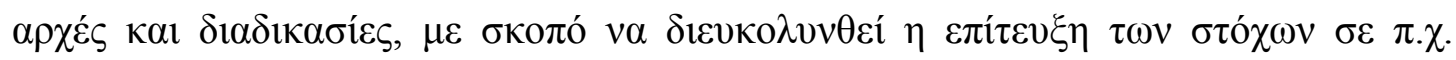

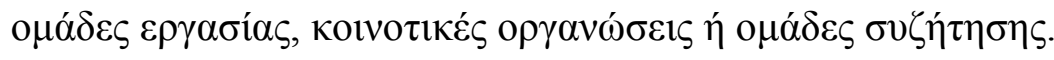

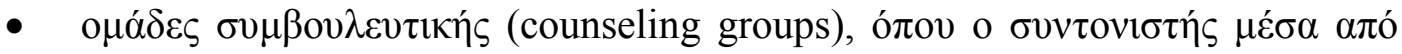

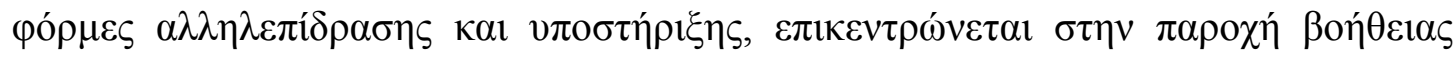




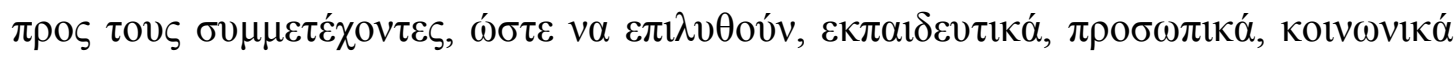

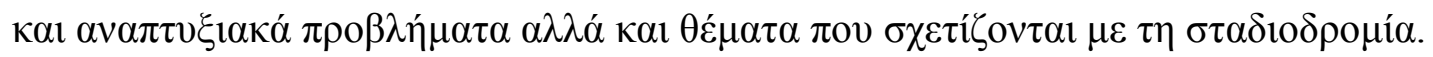

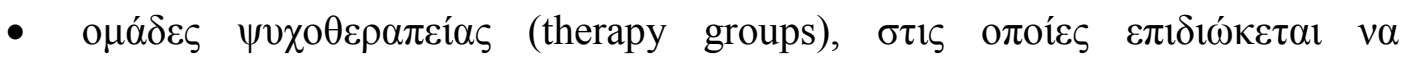

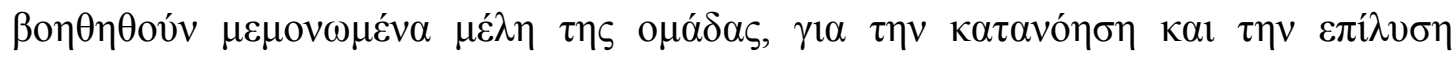

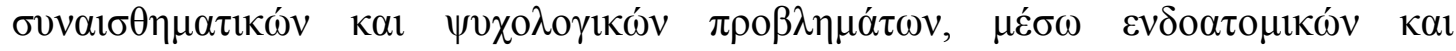

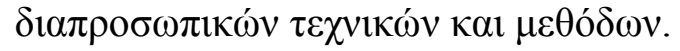

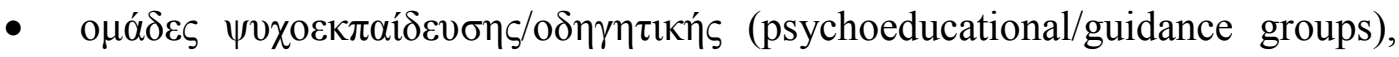

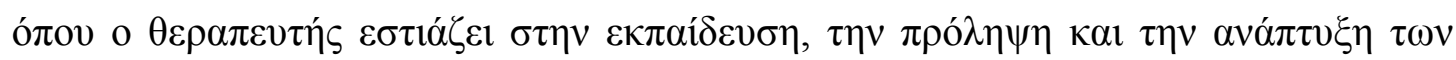

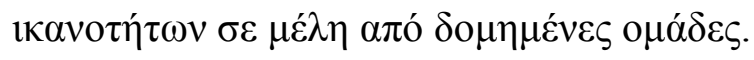

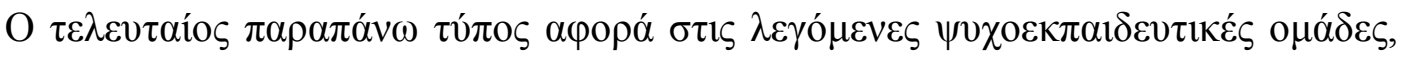

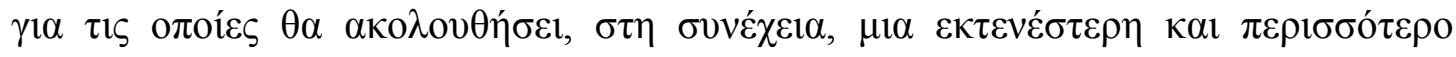

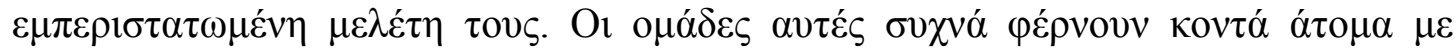

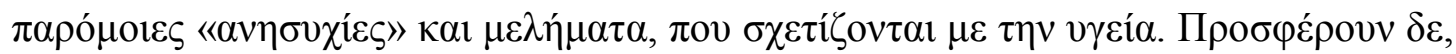

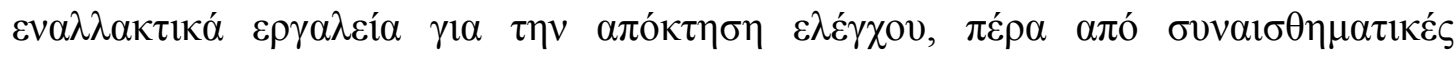

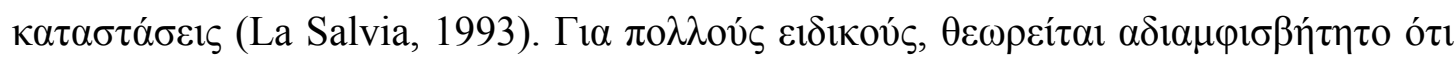

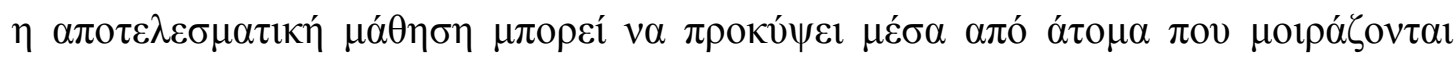

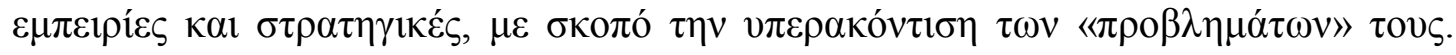

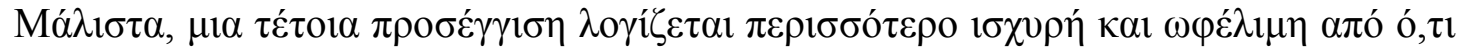

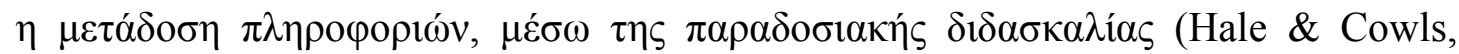
2015; Anderson 2001).

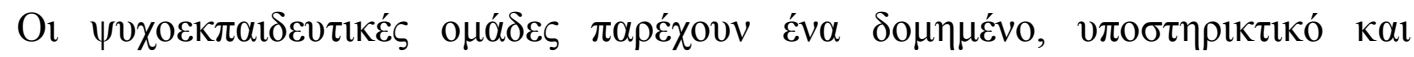

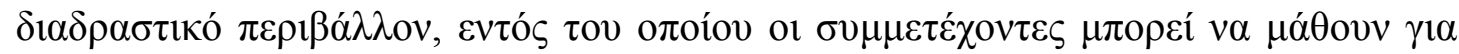

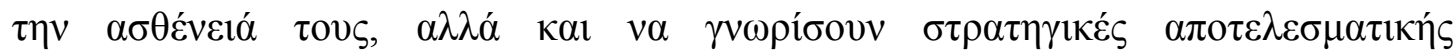

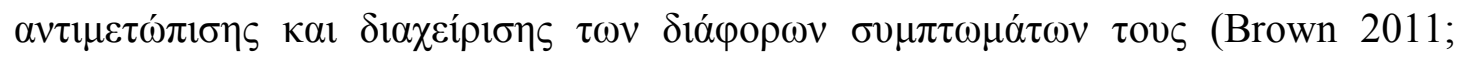
Pitschel-Walz et.al., 2009).

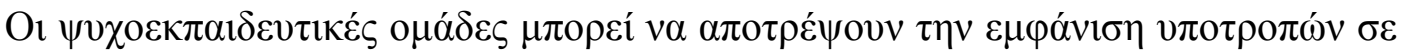

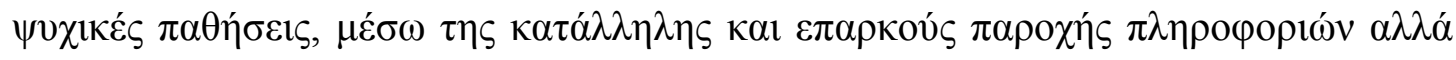

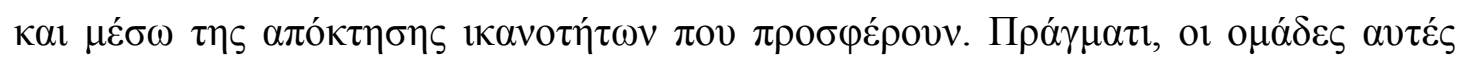

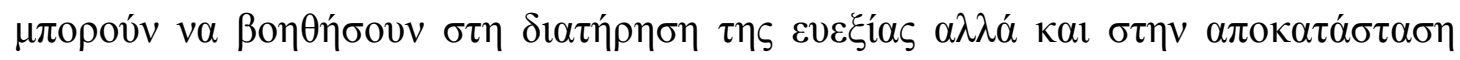

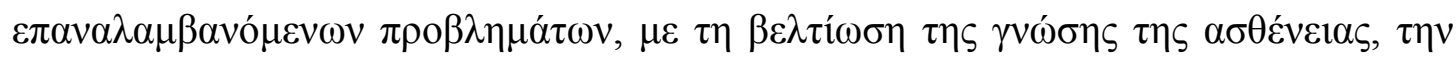

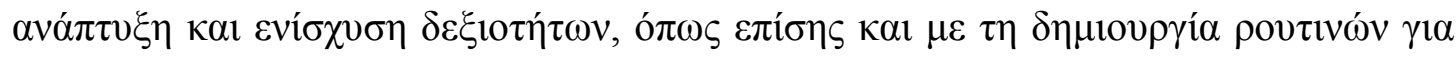

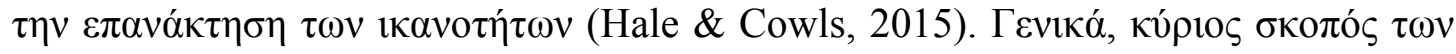

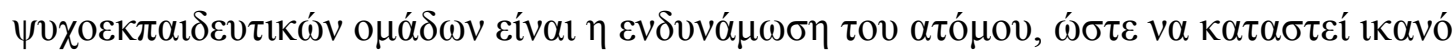




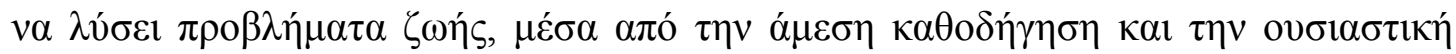

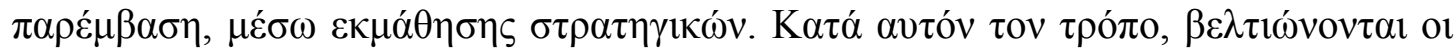

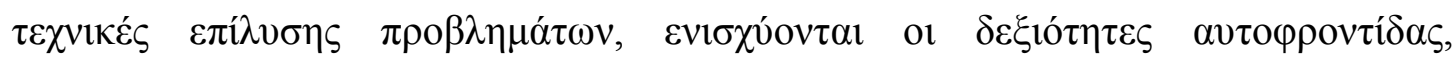

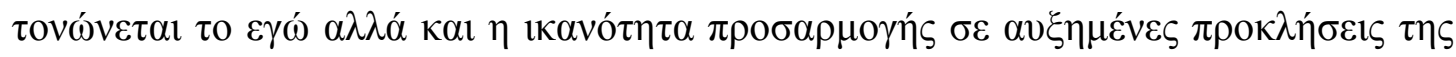

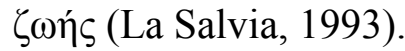

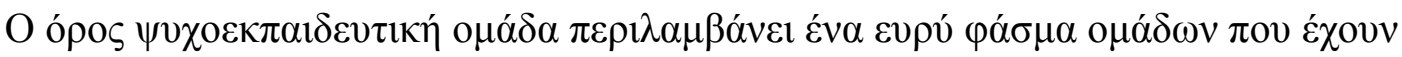

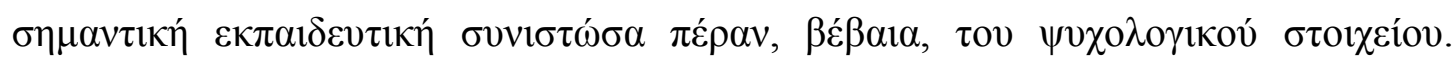

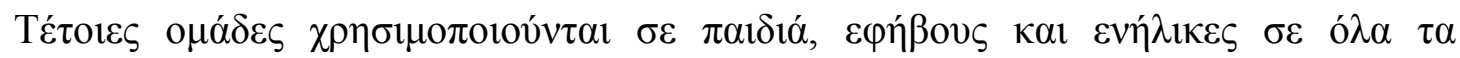

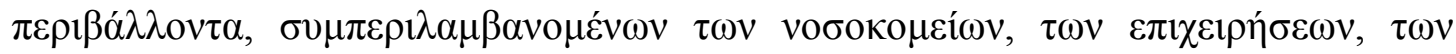

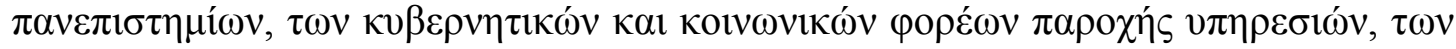

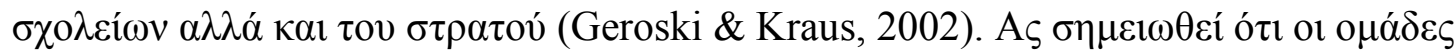

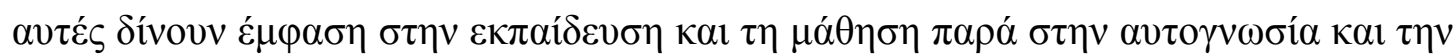

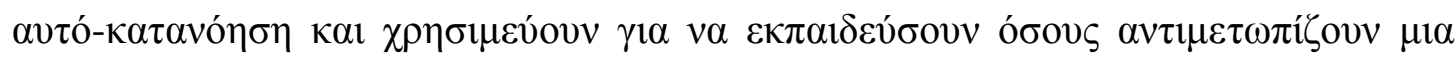

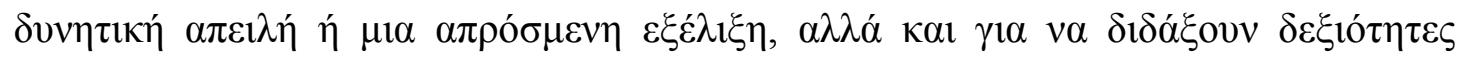

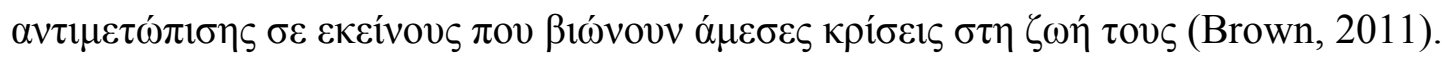

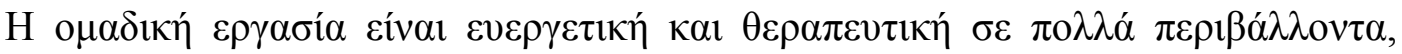

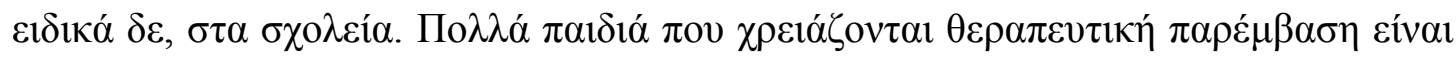

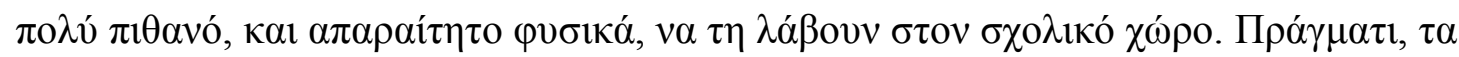

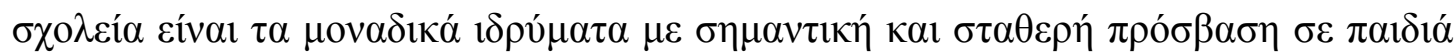

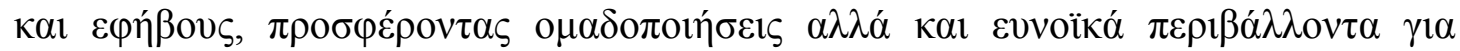

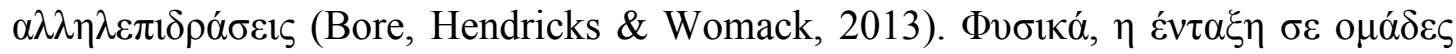

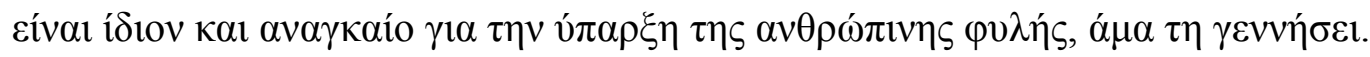

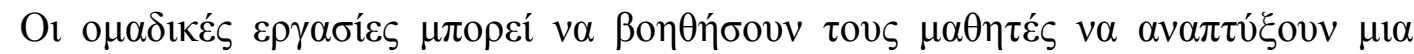

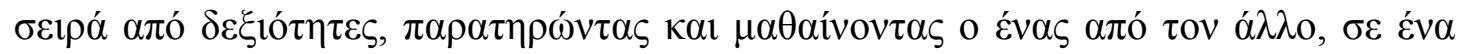

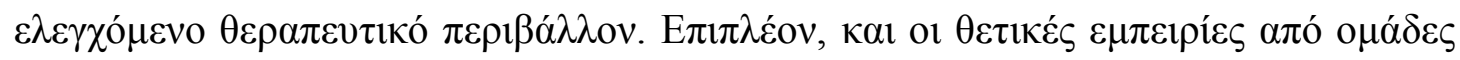

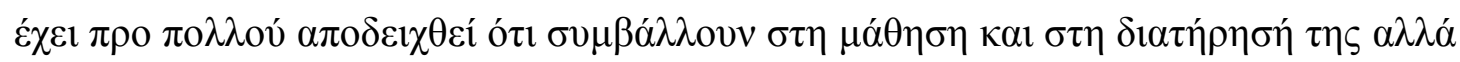

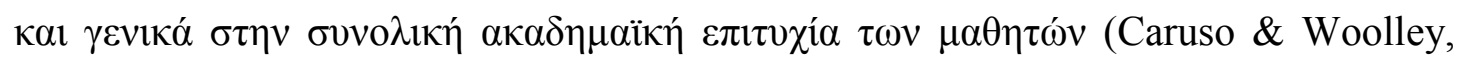

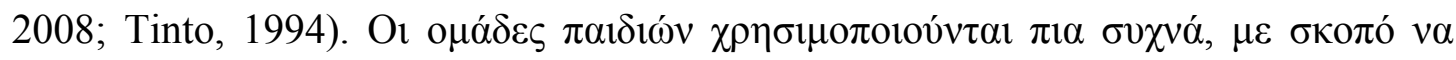

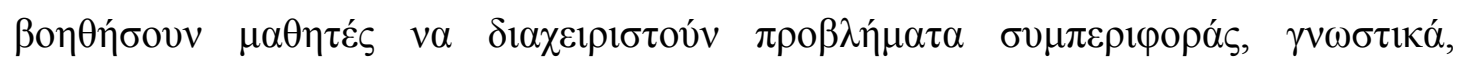

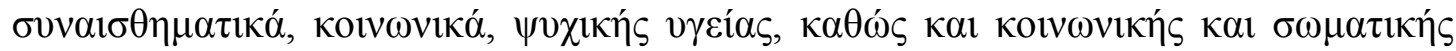

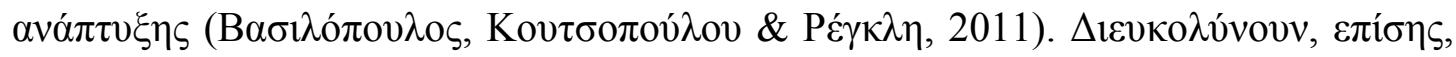

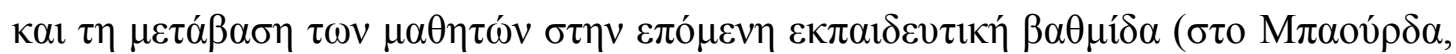




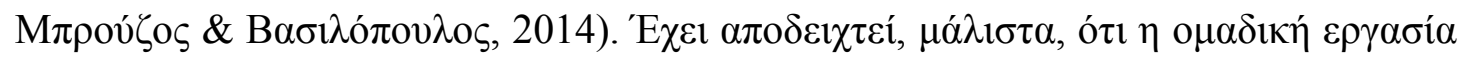

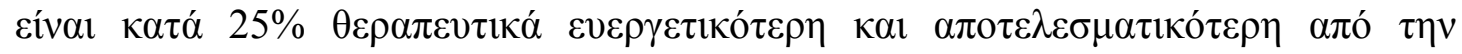

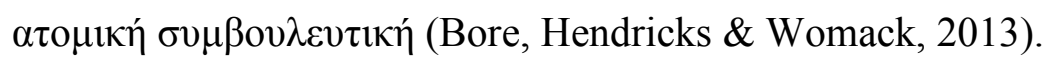

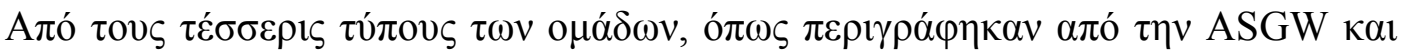

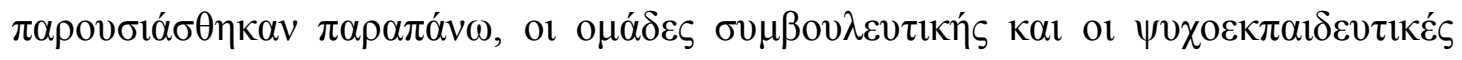

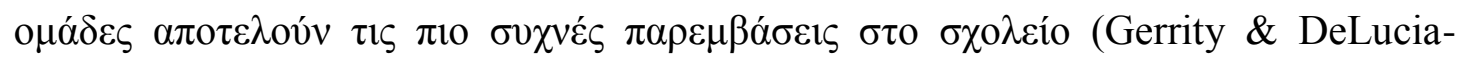

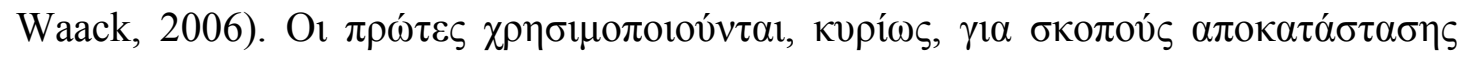

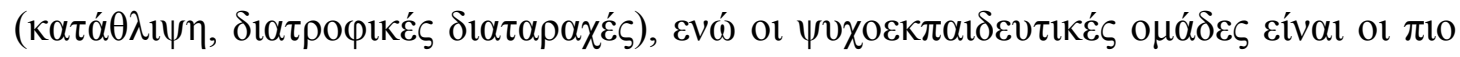

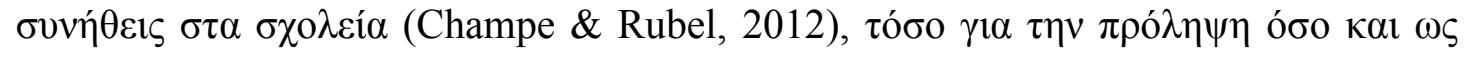

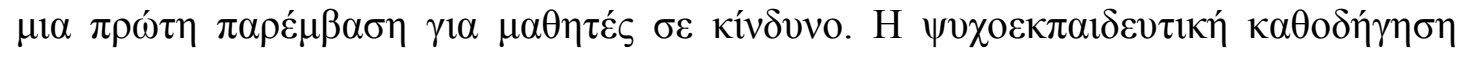

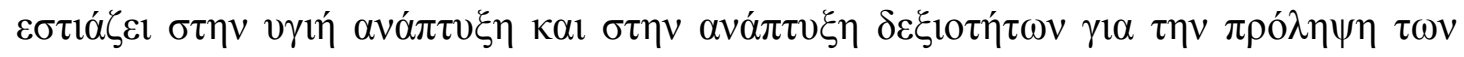

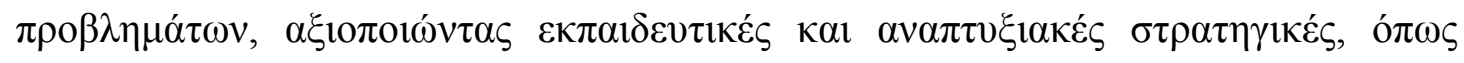

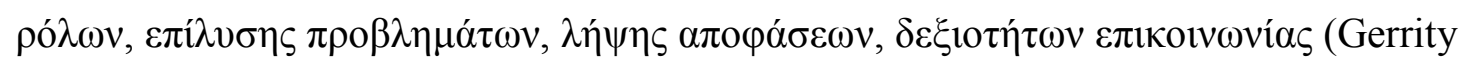

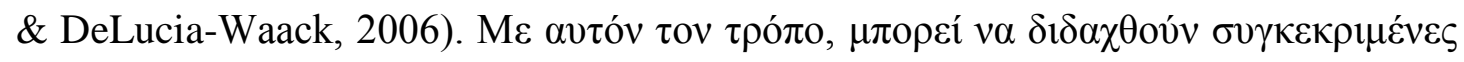

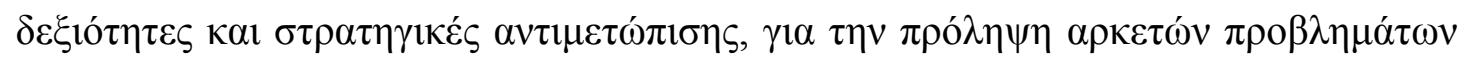

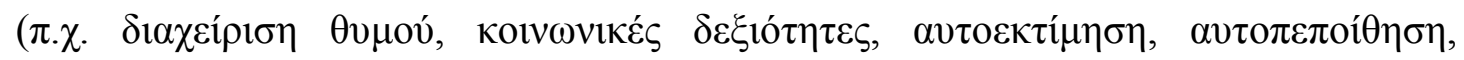

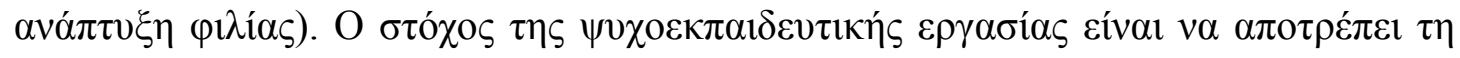

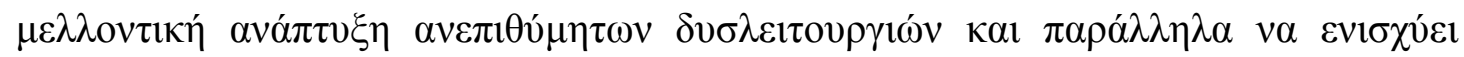

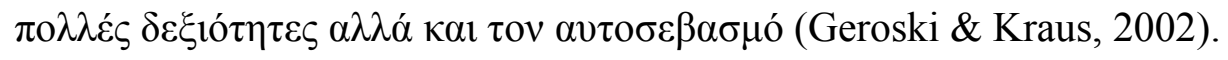

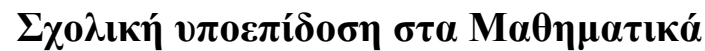

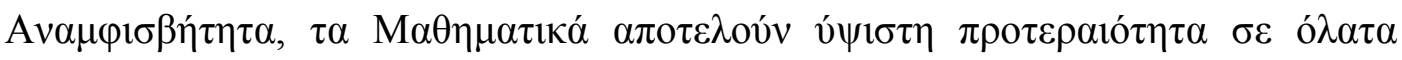

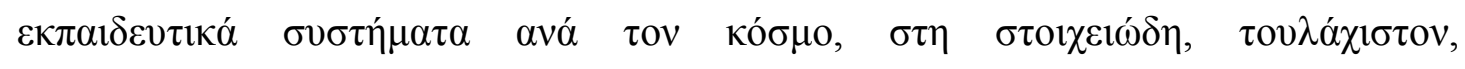

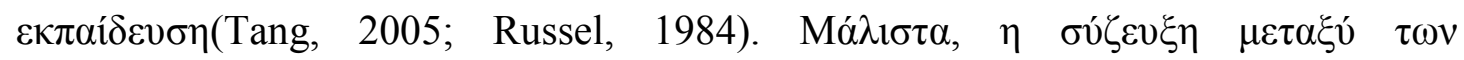

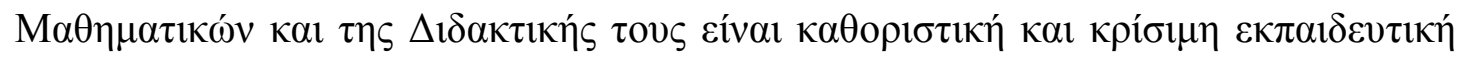

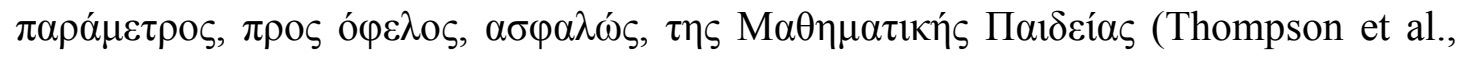
2014).

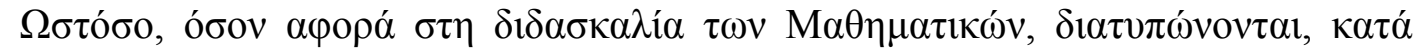

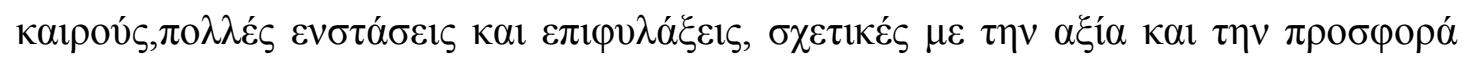

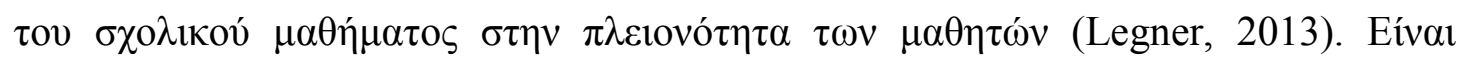

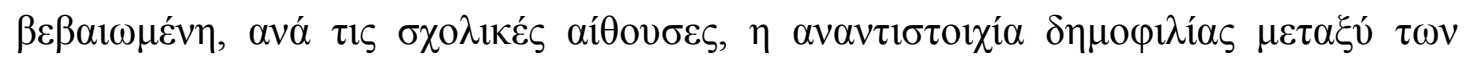

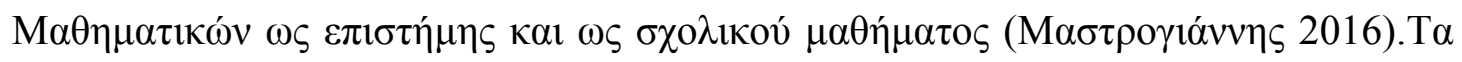

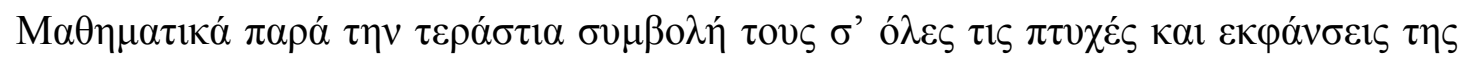




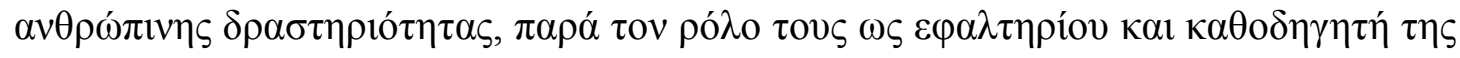

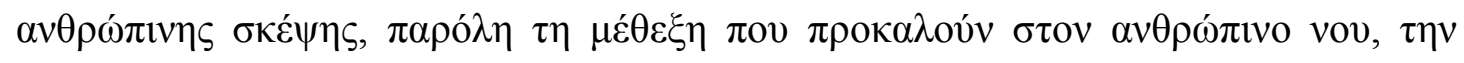

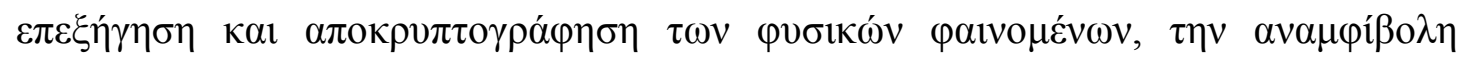

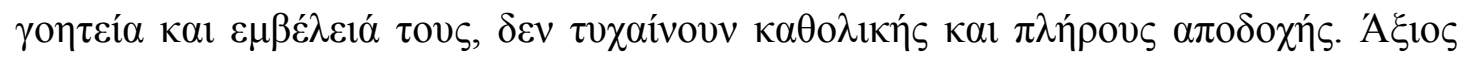

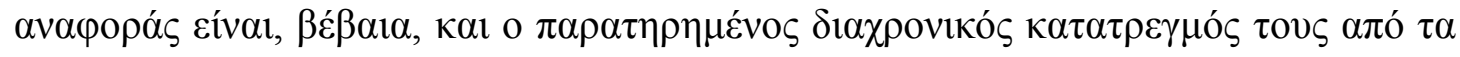

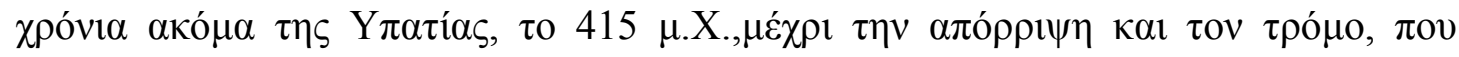

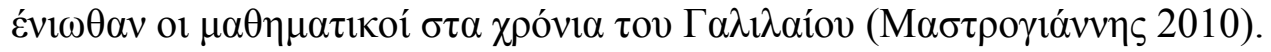

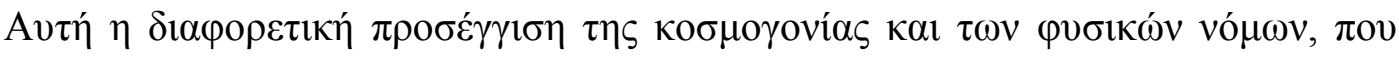

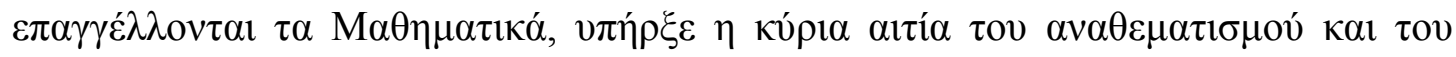

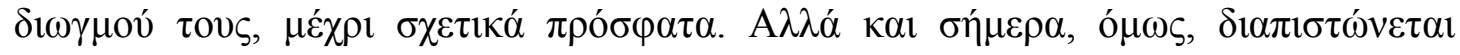

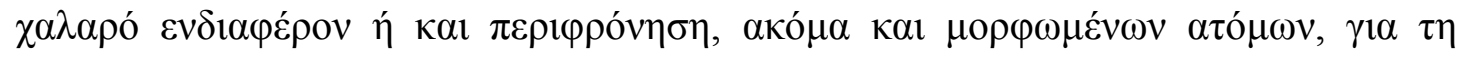

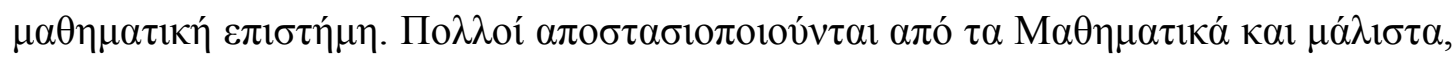

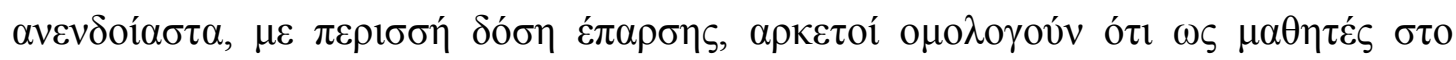

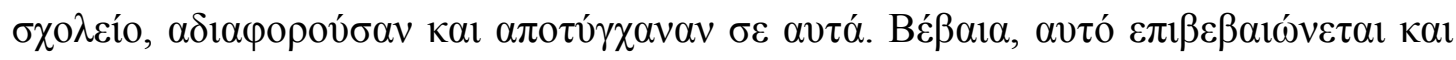

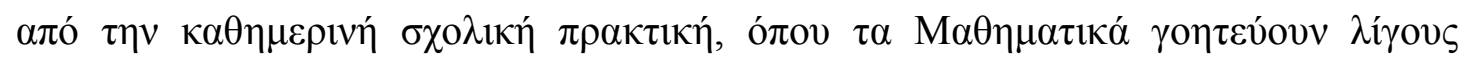

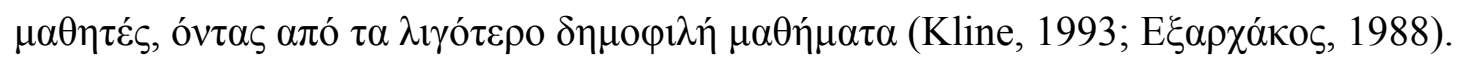

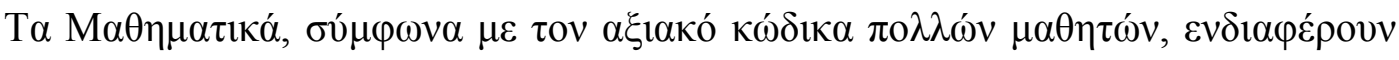

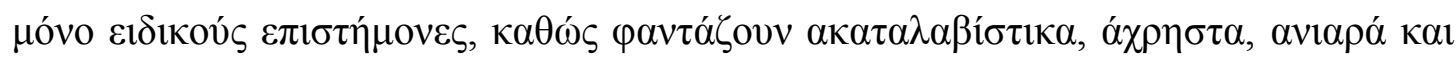

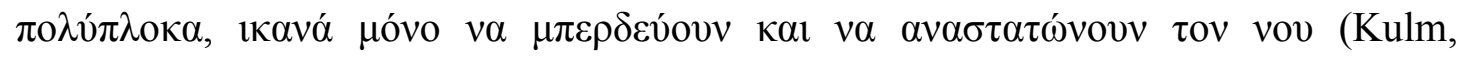

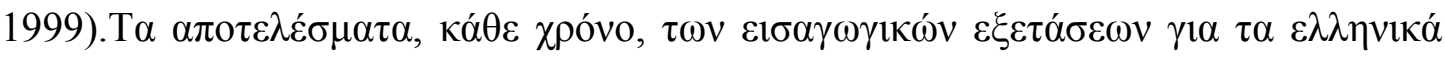

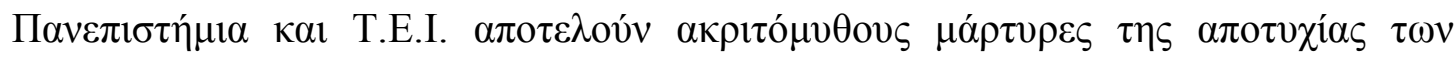

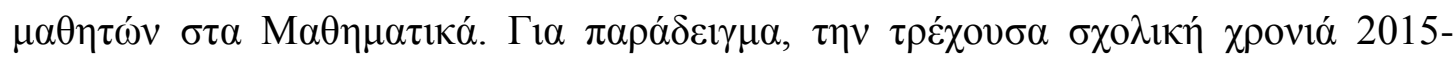

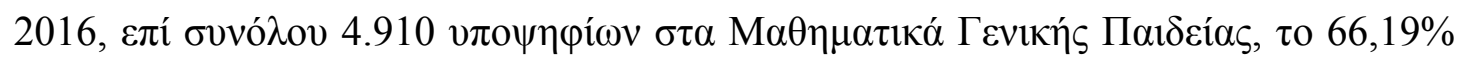

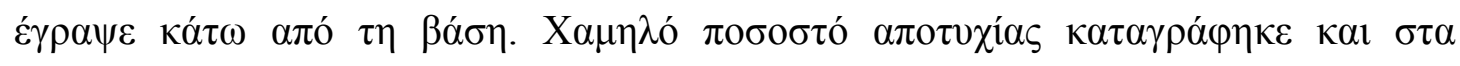

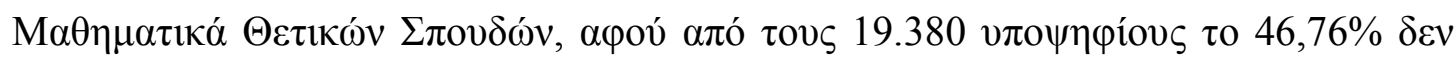

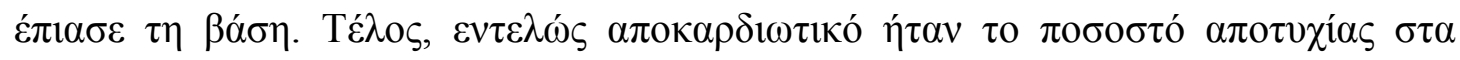

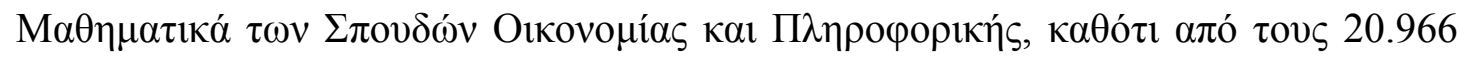

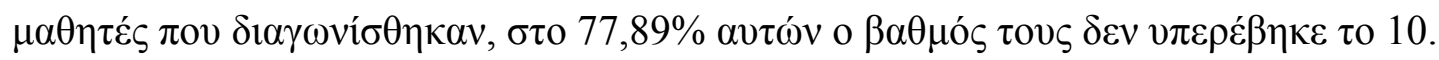

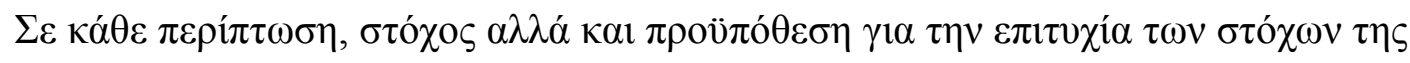

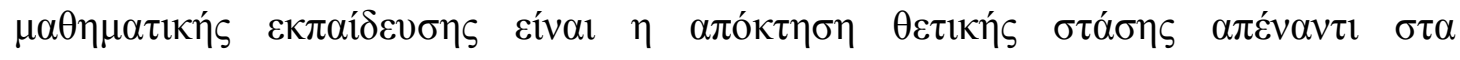

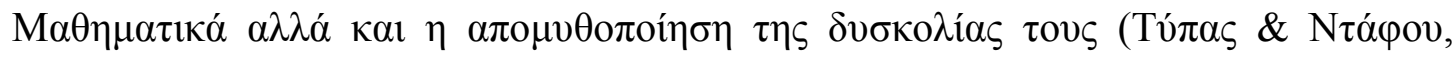

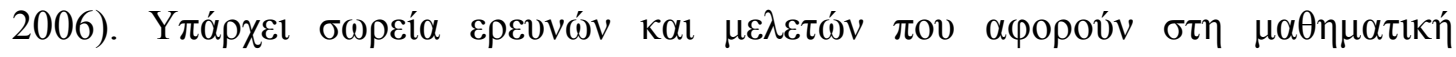




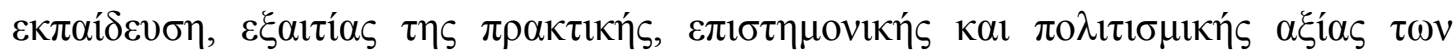

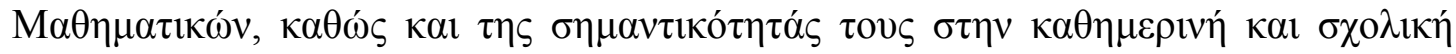

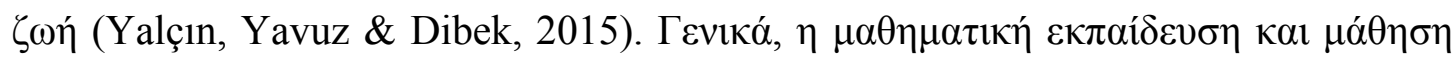

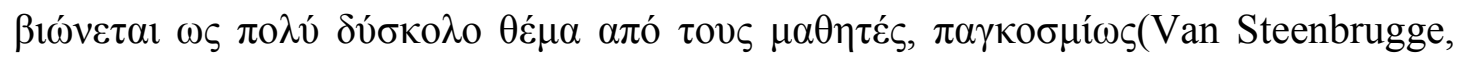

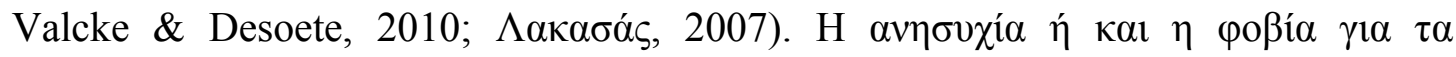

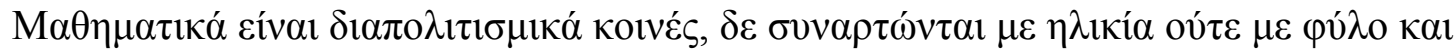

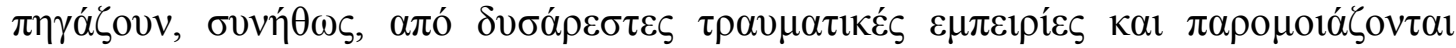

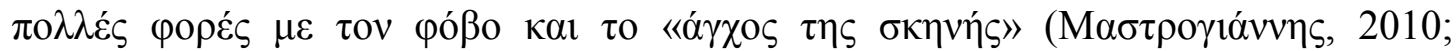

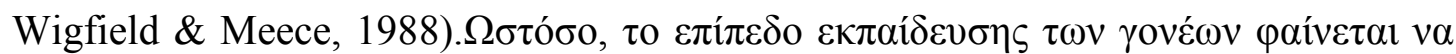

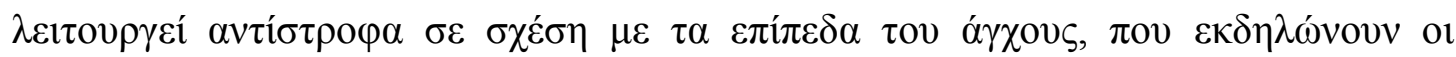

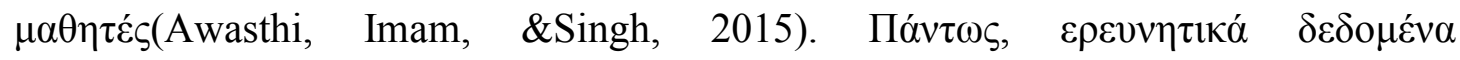

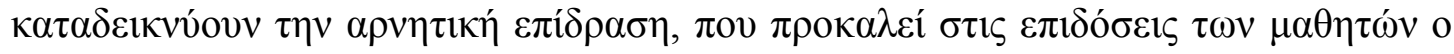

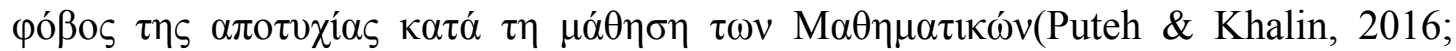
Pantziara \& Philippou, 2015).

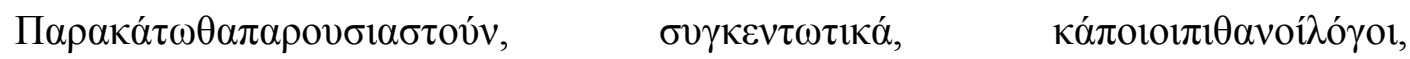

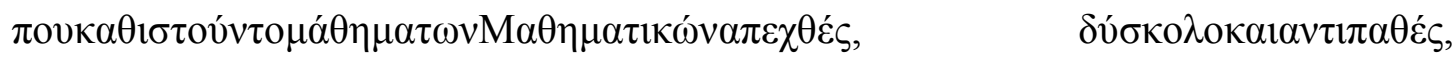

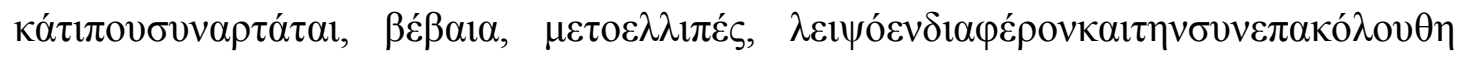

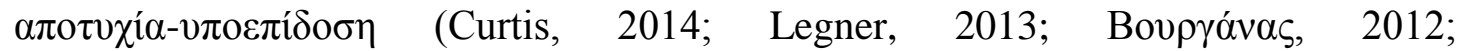

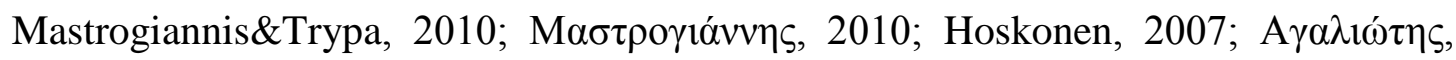

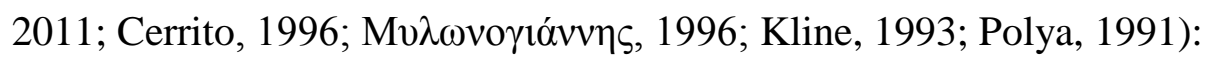

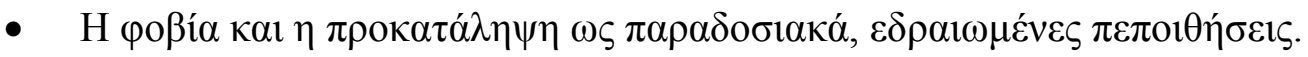

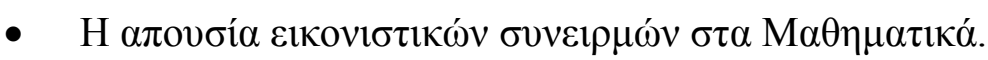

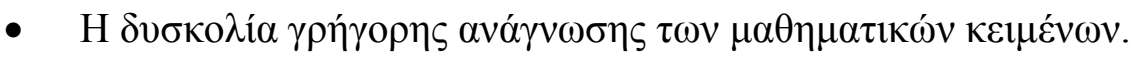

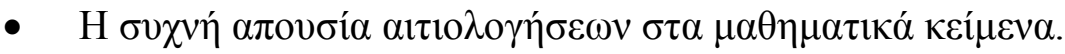

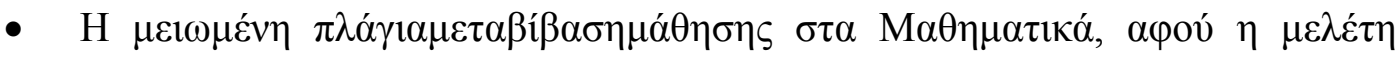

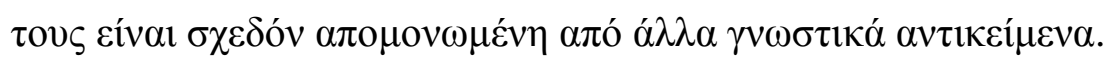

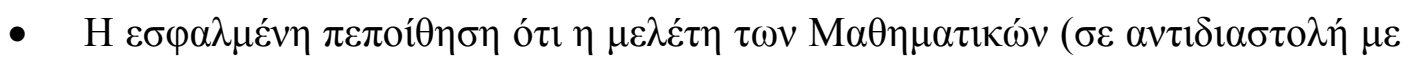
$\tau \eta \nu$ A

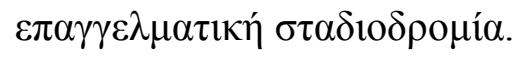

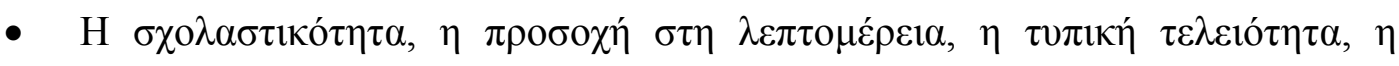

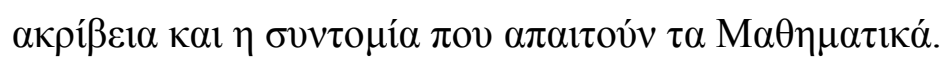


- H

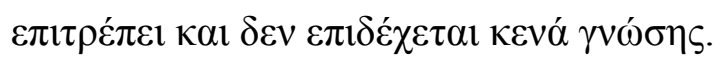

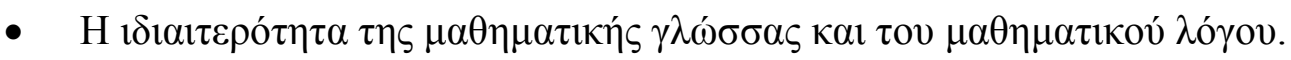

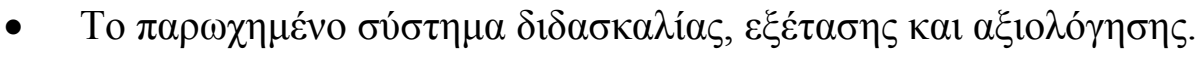

- $\mathrm{H} \pi \lambda \eta \theta \omega ́ \rho \alpha \tau\rceil \zeta \delta \delta \alpha \kappa \tau \varepsilon \dot{\alpha} \alpha v^{\prime} \lambda \eta \varsigma$.

- H $\pi 010 ́ \tau \eta \tau \alpha \tau \omega \nu \varepsilon \gamma \chi \varepsilon i \rho 1 \delta i ́ \omega v$.

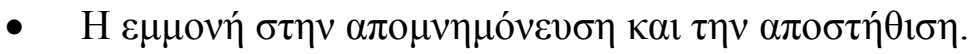

- H

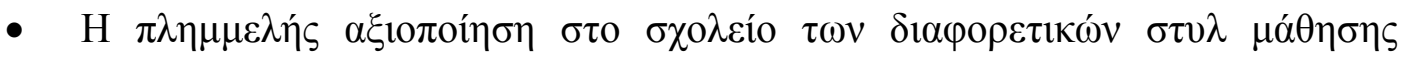

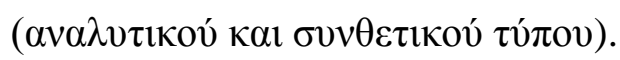

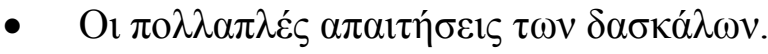

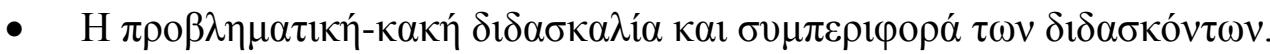

- $\mathrm{H} « \alpha \nu \tau \imath \delta 1 \kappa i ́ \alpha » \delta \alpha \sigma \kappa \alpha ́ \lambda \omega v-\kappa \alpha \theta \eta \gamma \eta \tau \omega ́ v$.

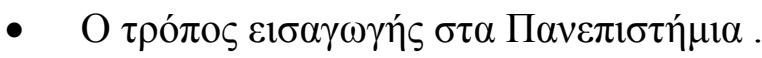

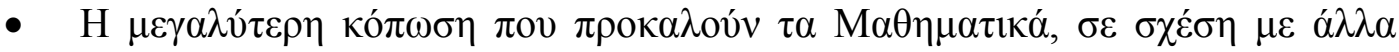
$\mu \alpha \theta \dot{\eta} \mu \alpha \tau \alpha$.

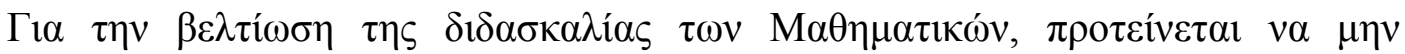

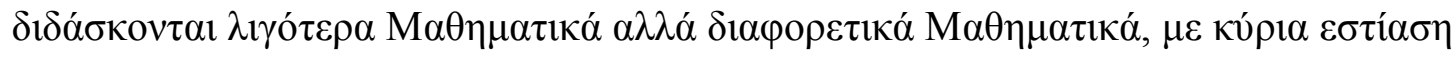

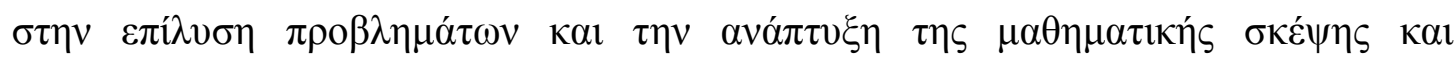

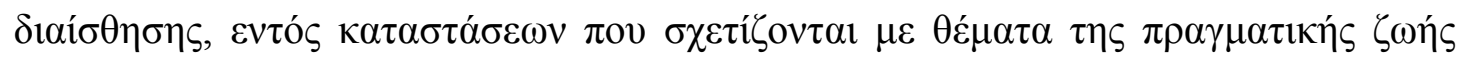

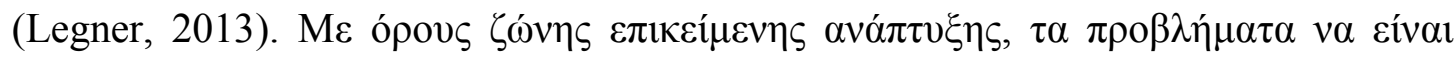

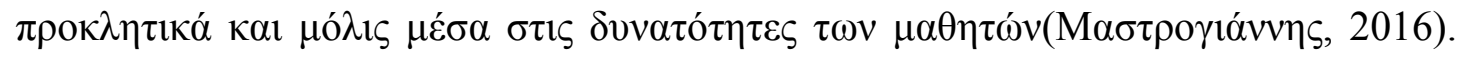

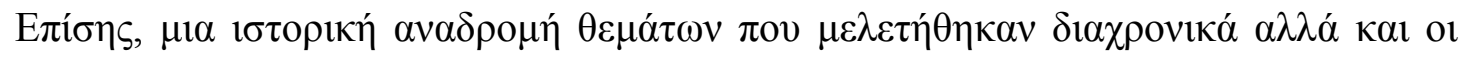

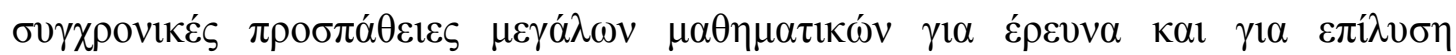

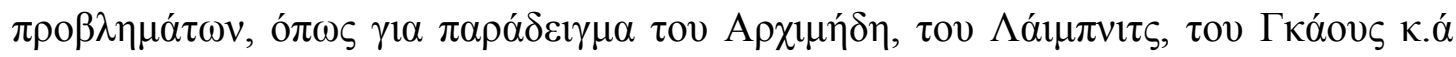

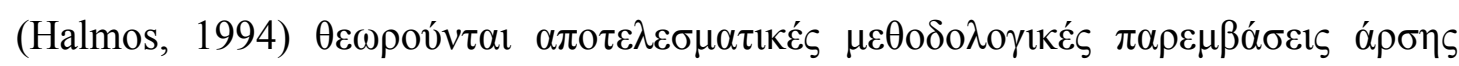

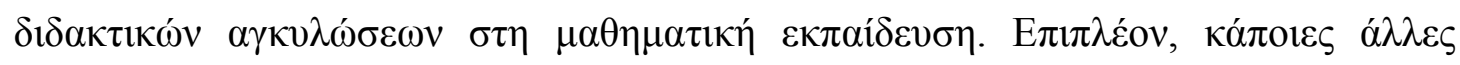

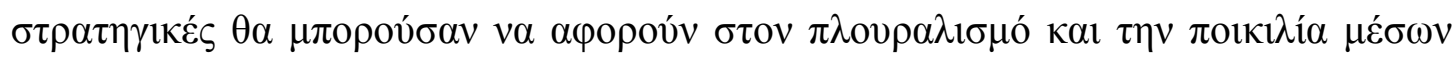

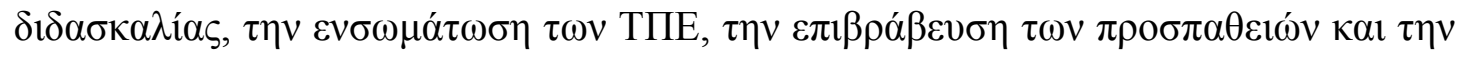

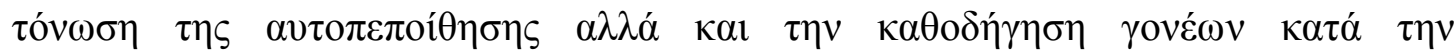

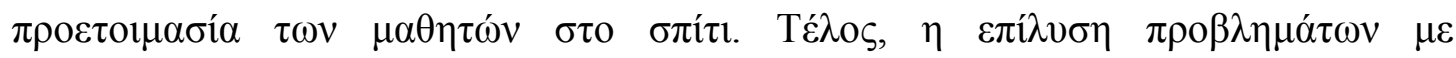

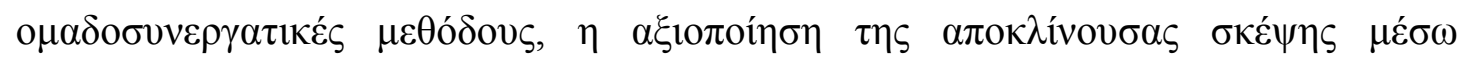

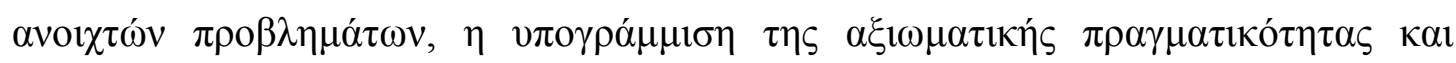




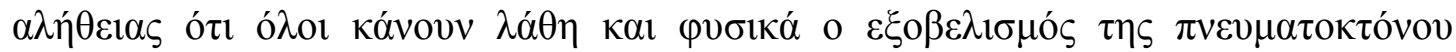

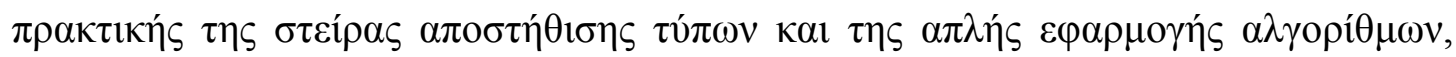

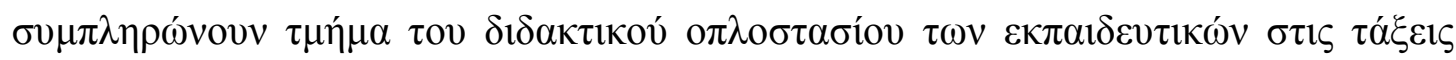

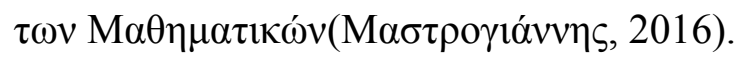

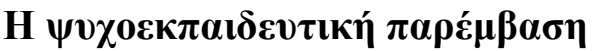

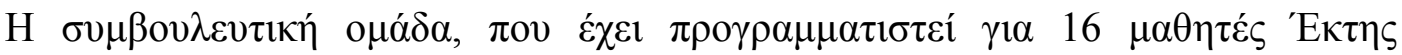

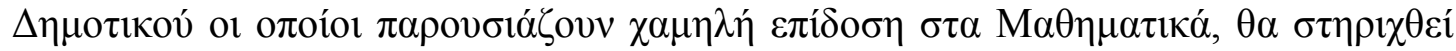

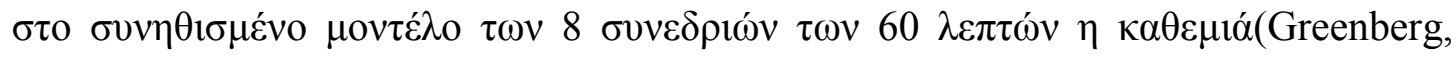

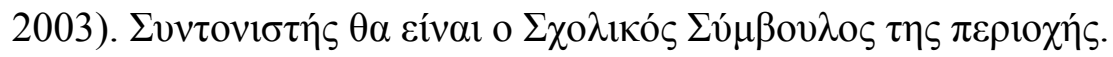

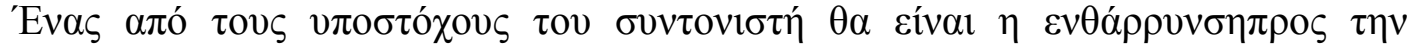

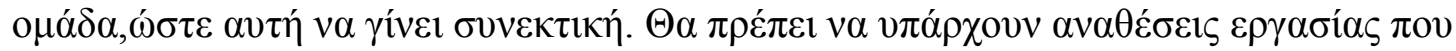

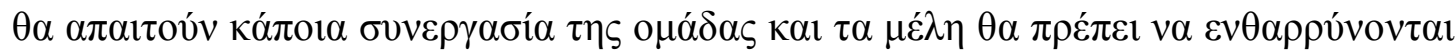

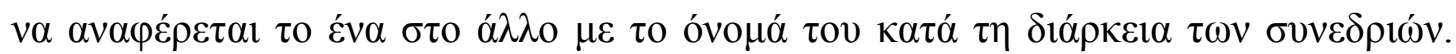

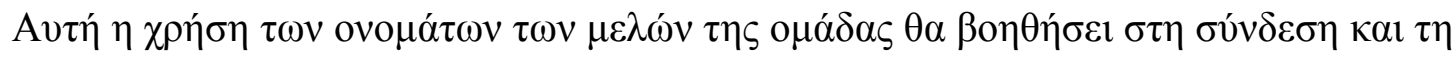

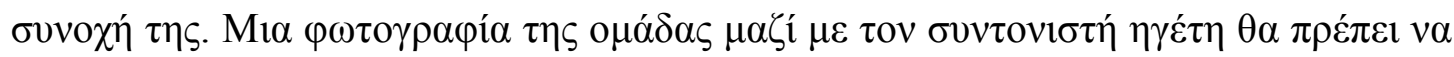

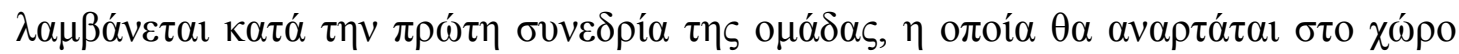

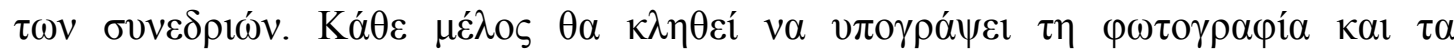

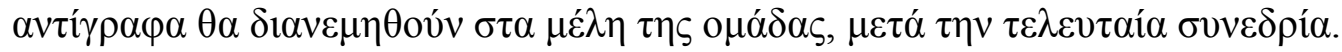

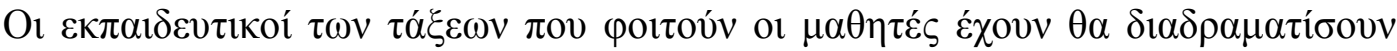

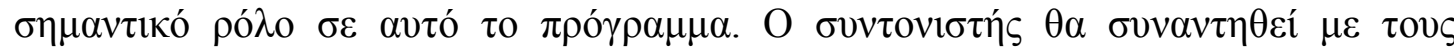

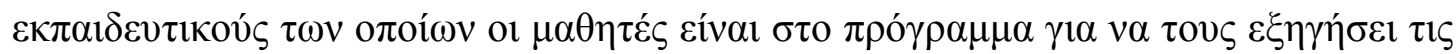

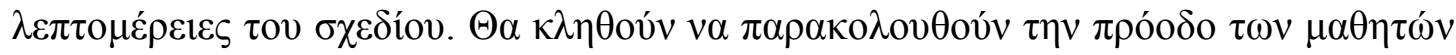

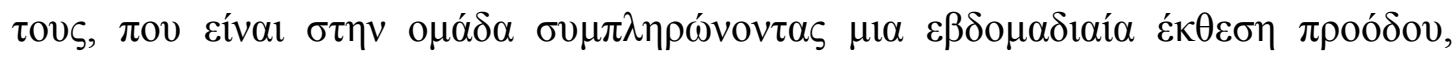

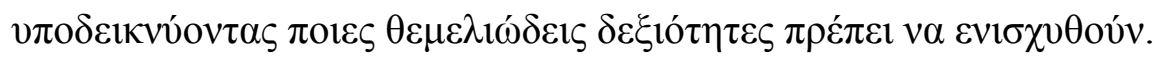

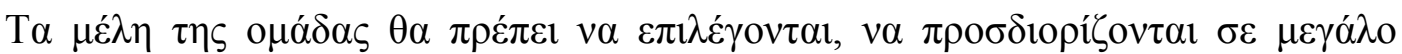

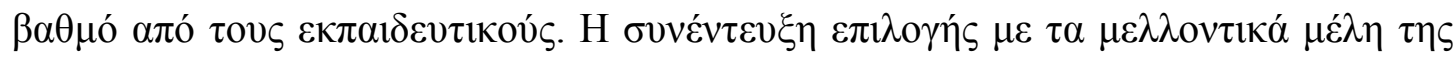

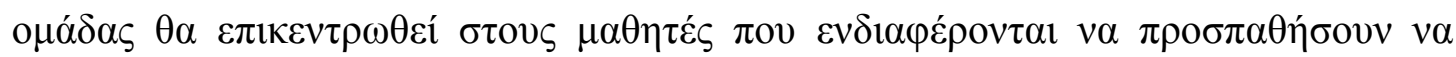

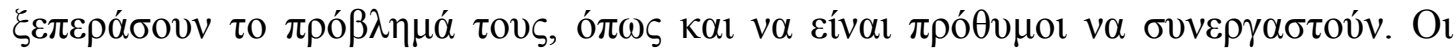

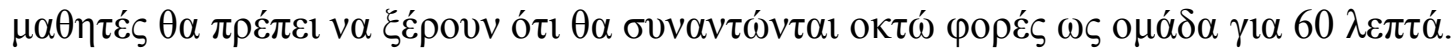

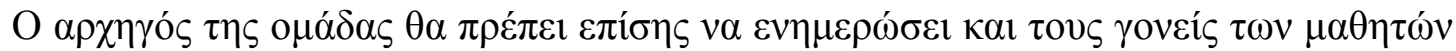




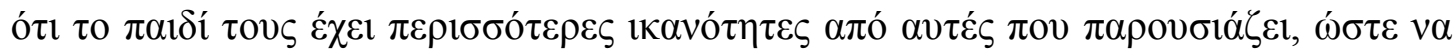

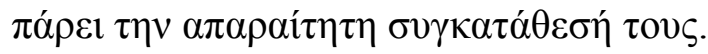

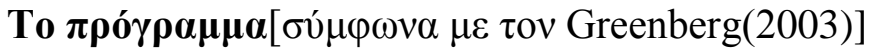

- $\quad \Pi \lambda \eta \theta v \sigma \mu o ́ \varsigma: ~ M \alpha \theta \eta \tau \varepsilon ́ \varsigma \Sigma \tau^{\prime} \Delta \eta \mu о \tau 1 \kappa o v ́$.

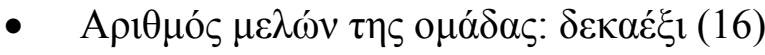

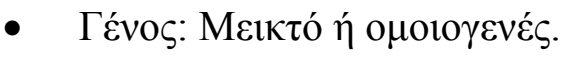

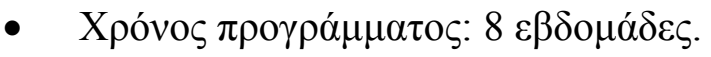

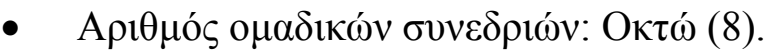

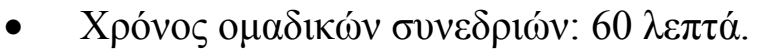

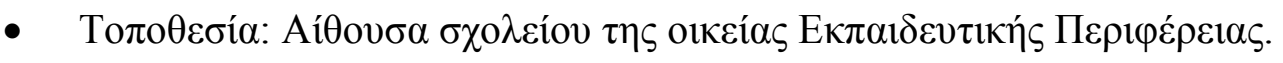

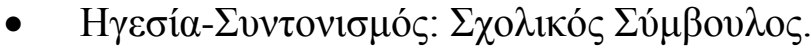

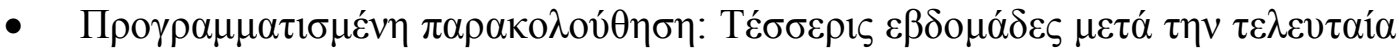

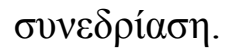

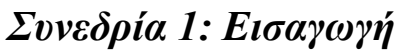

\section{$\underline{\Sigma \tau o ́} \chi 01$}

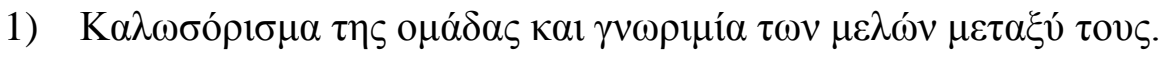

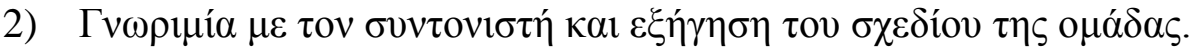

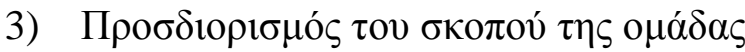

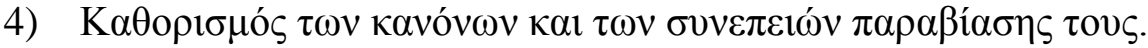

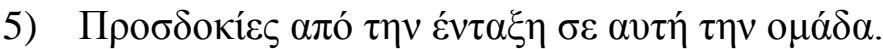

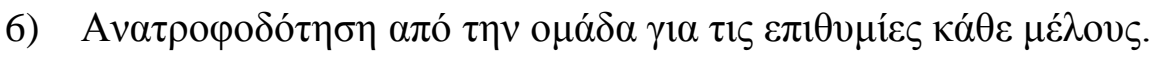

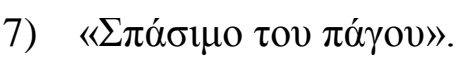

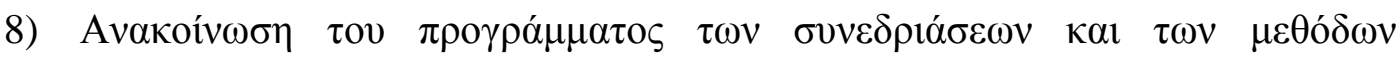

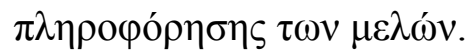

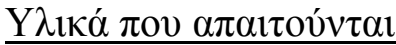

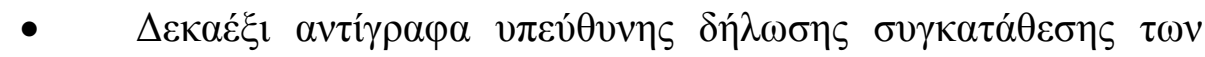

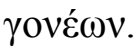

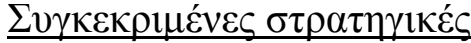

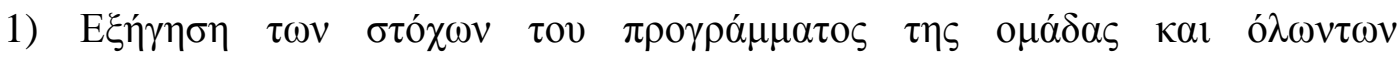

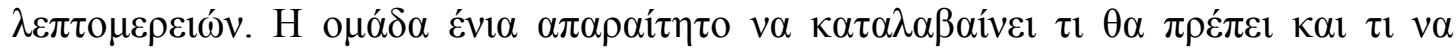




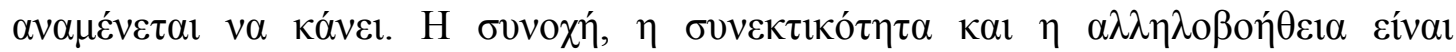

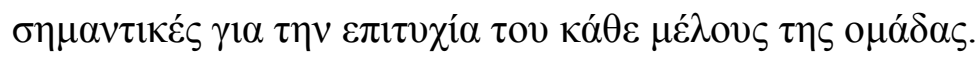

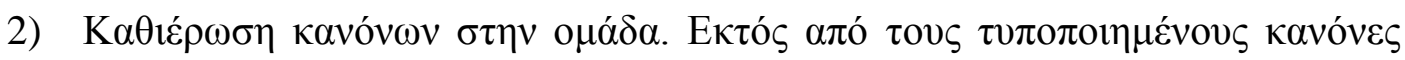

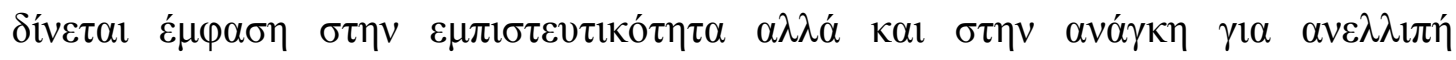

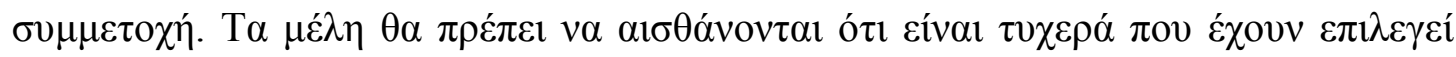

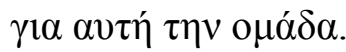

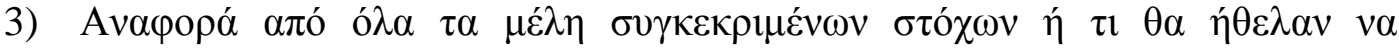

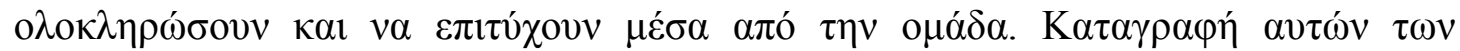

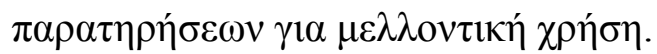

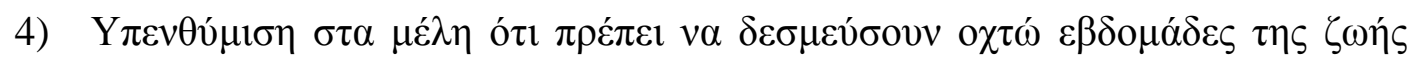

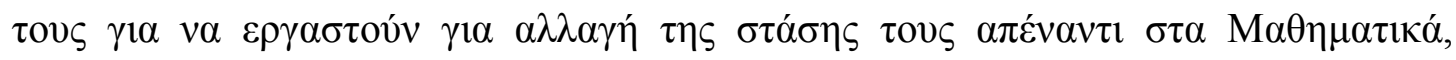

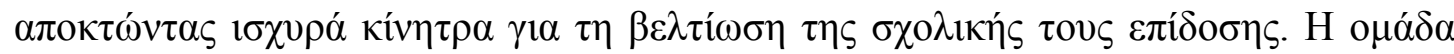

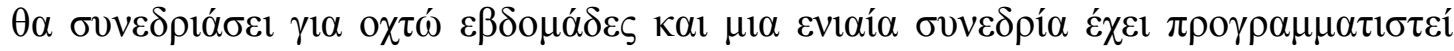

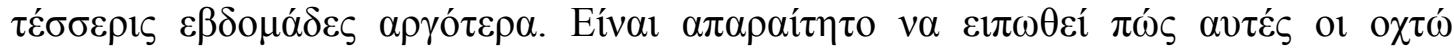

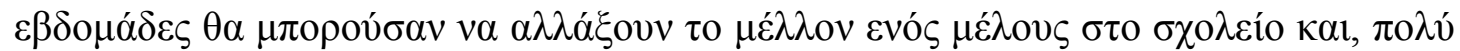

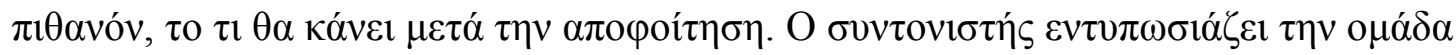

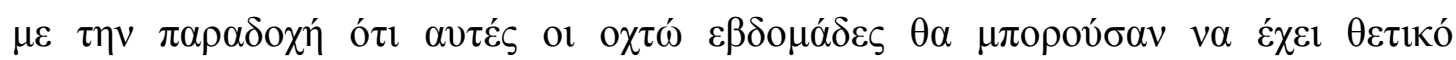

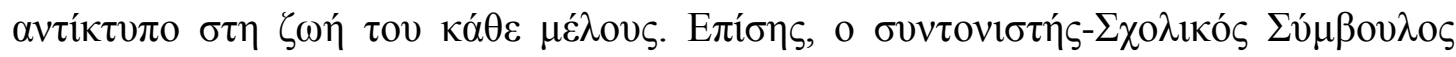

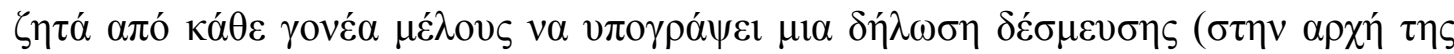

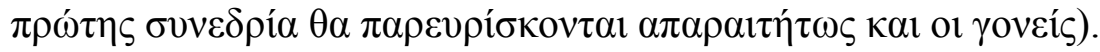

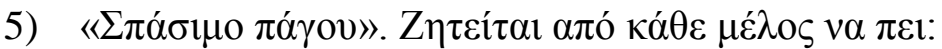

i. Поюю $\theta \alpha \lambda \varepsilon \dot{\varepsilon} \gamma \alpha \tau \varepsilon$ ó

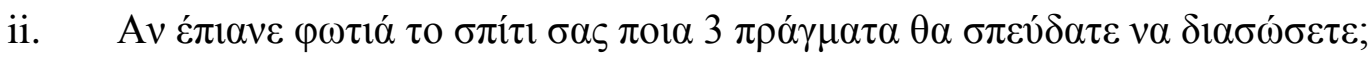

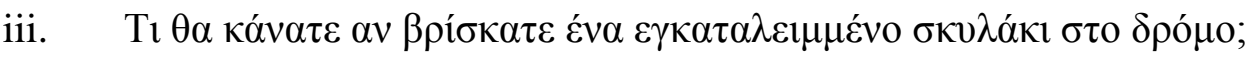

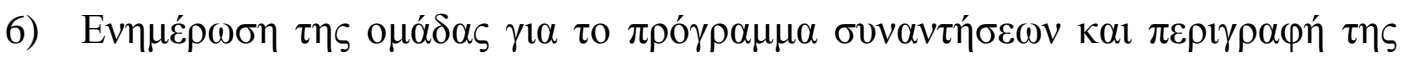

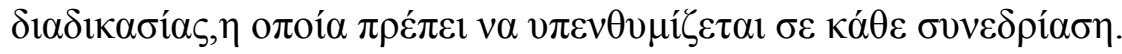

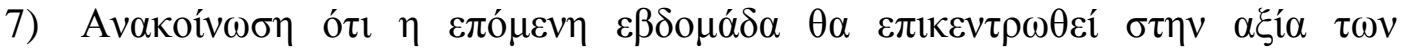

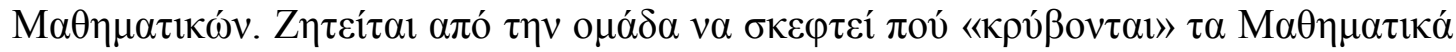

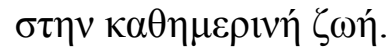

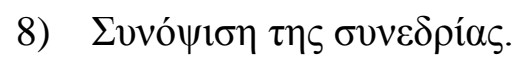

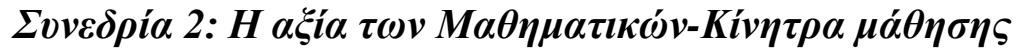

\section{$\underline{\Sigma \tau o ́} \chi 01$}




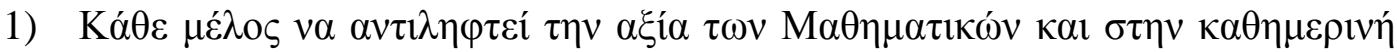
$\zeta \omega \eta ́$.

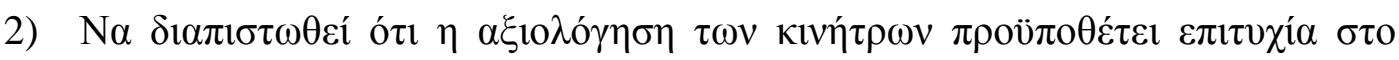
$\sigma \chi 0 \lambda \varepsilon i ́ o$.

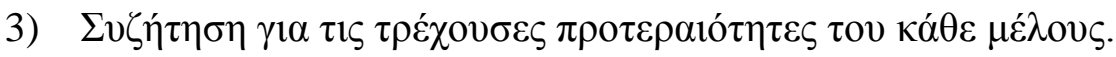

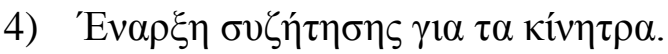

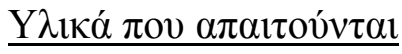

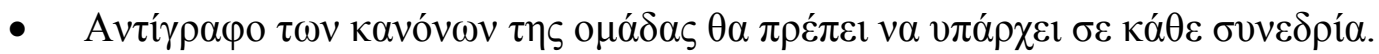

$\underline{\Sigma v \gamma \kappa \varepsilon \kappa \rho \mu \varepsilon^{\prime} v \varepsilon \zeta \sigma \tau \rho \alpha \tau \eta \gamma 1 \kappa \varepsilon ́ \varsigma}$

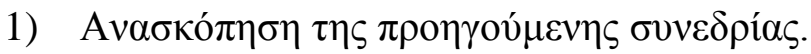

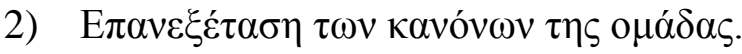

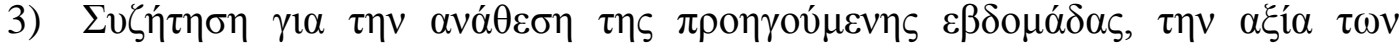

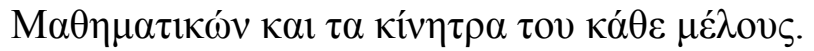

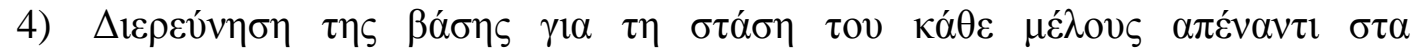

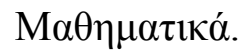

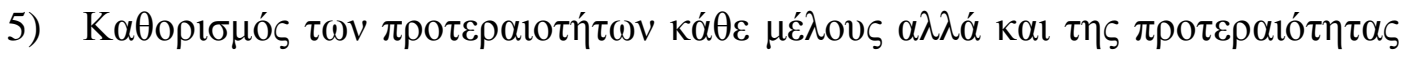

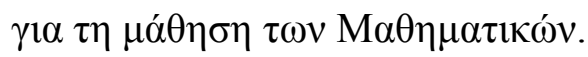

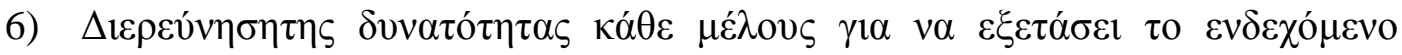

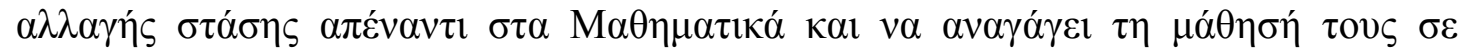

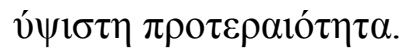

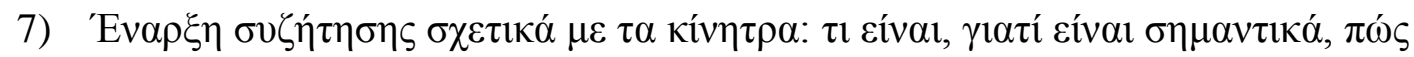

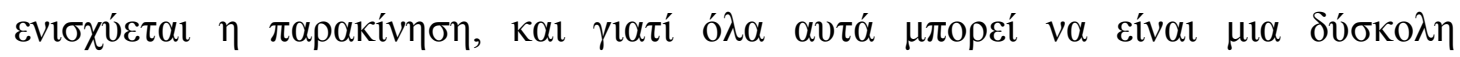

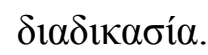

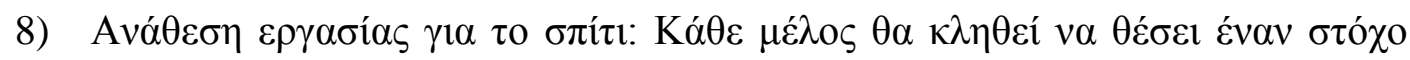

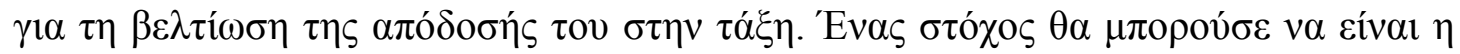

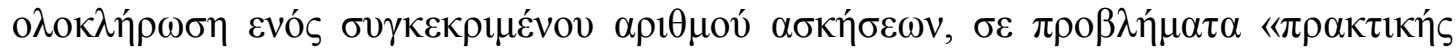

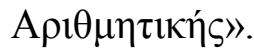

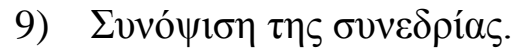

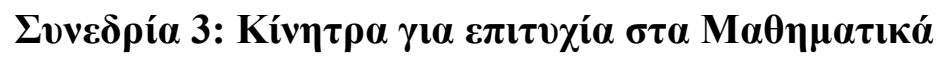

\section{$\underline{\Sigma \tau o ́} \chi 01$}

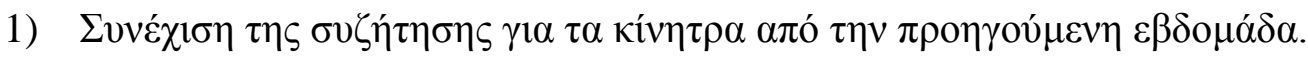

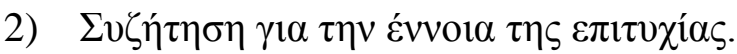




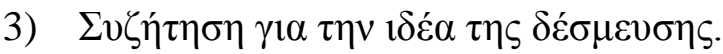

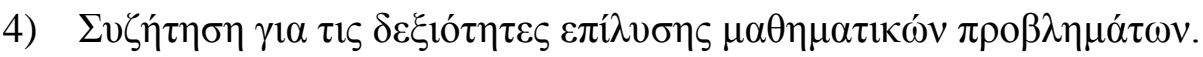

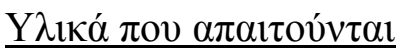

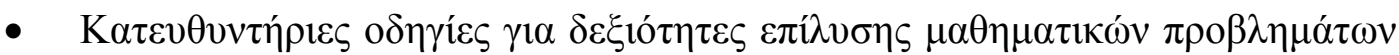

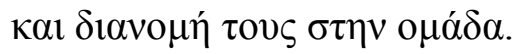

\section{$\underline{\Sigma v \gamma \kappa \varepsilon \kappa \rho \mu \varepsilon ́ v \varepsilon \varsigma \sigma \tau \rho \alpha \tau \eta \gamma \kappa \varepsilon \varepsilon \varsigma}$}

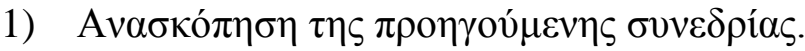

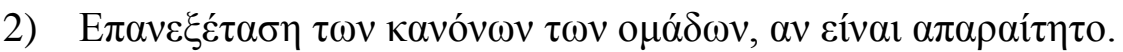

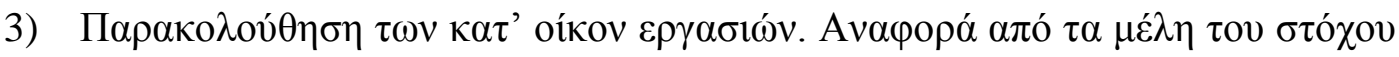

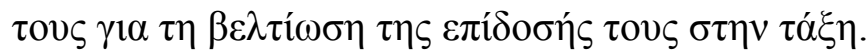

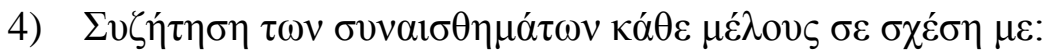

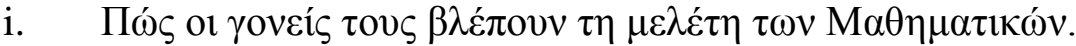

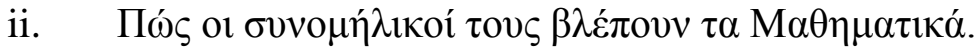

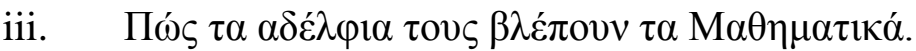

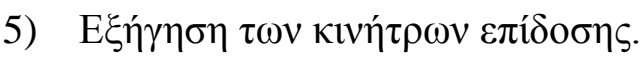

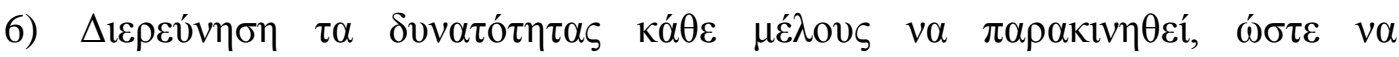

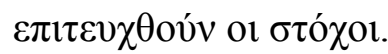

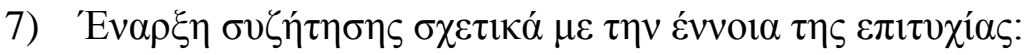

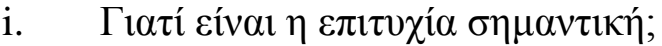

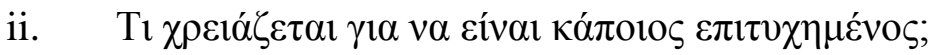

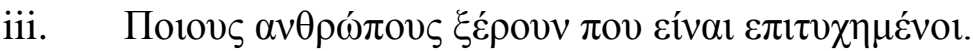

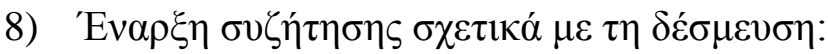

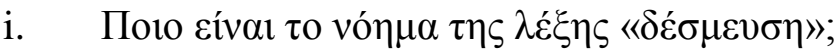

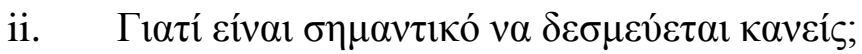

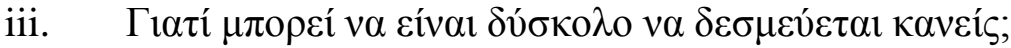

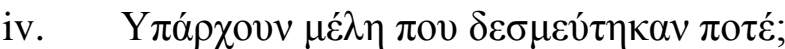

v. $\quad A v v \alpha 1, \sigma \varepsilon \tau \imath$;

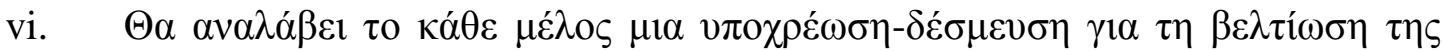

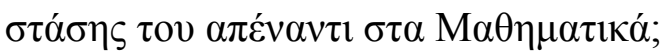

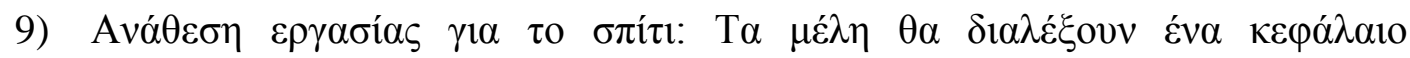

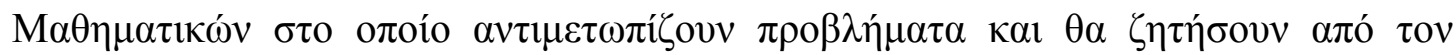

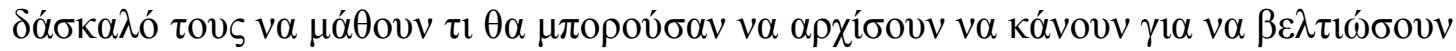




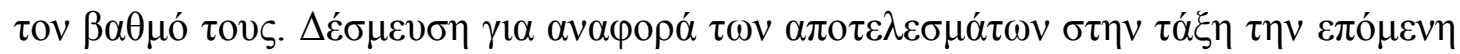
$\varepsilon \beta \delta о \mu \alpha ́ \delta \alpha$.

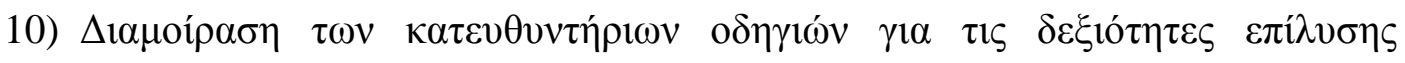

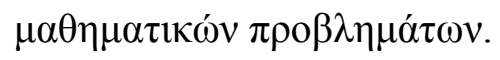

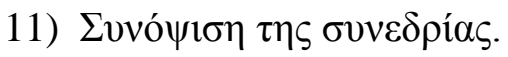

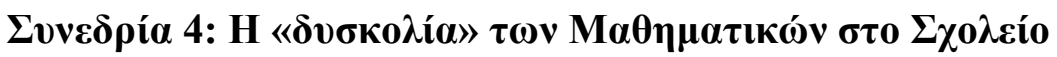

\section{$\underline{\Sigma \tau o ́} \chi 01$}

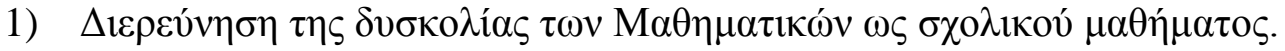

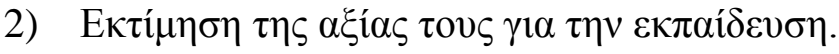

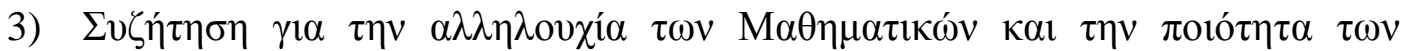

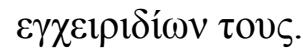

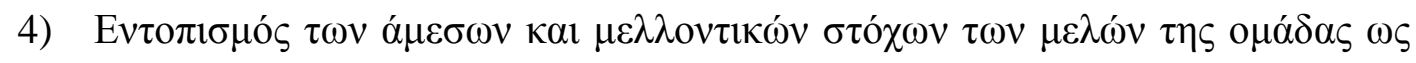

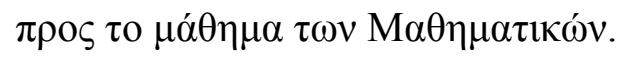

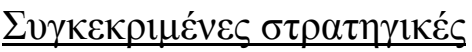

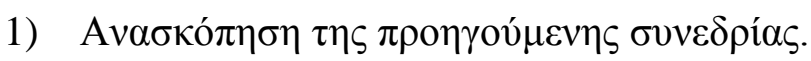

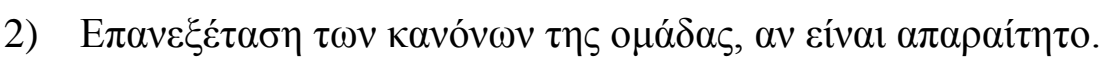

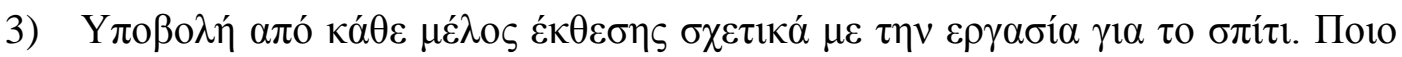

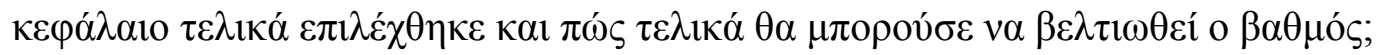

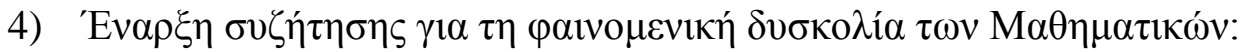

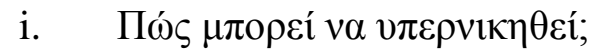

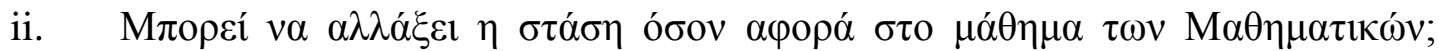

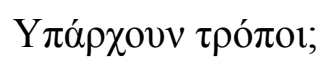

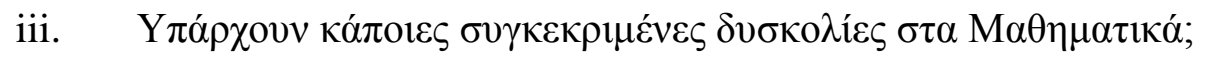

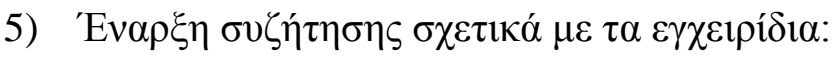

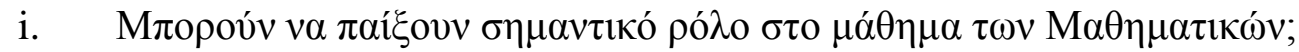

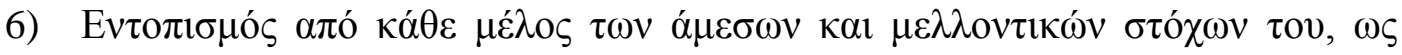

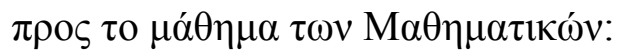

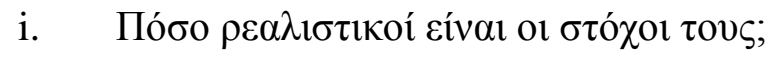

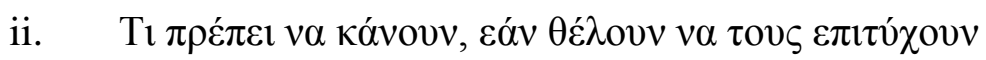




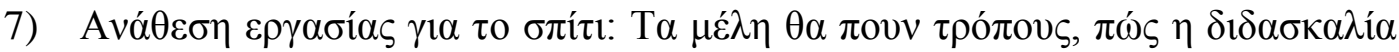

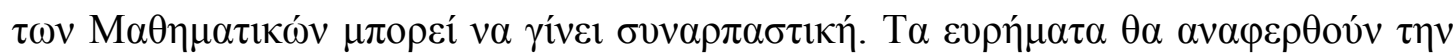

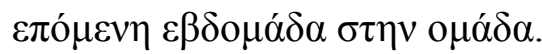

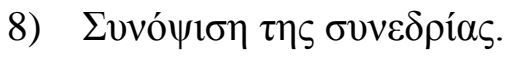

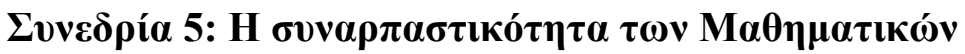

\section{$\underline{\Sigma \tau o ́} \chi 01$}

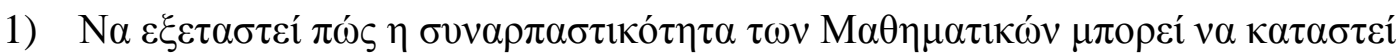

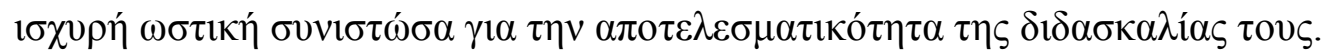

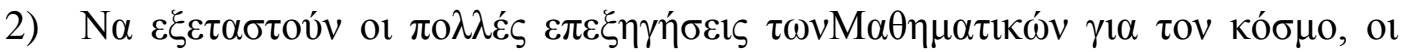

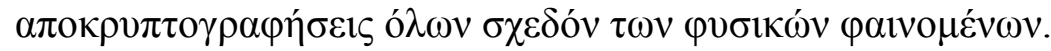

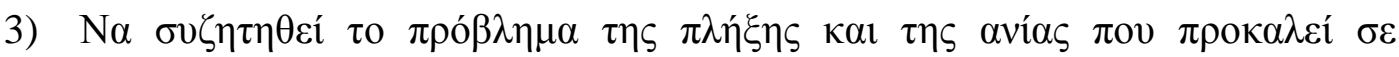

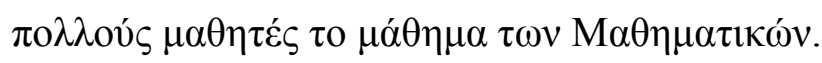

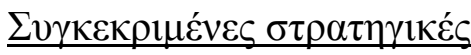

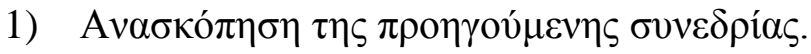

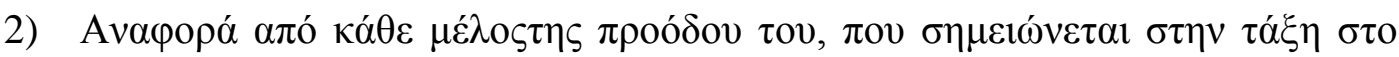
$\mu \alpha \dot{\theta \eta \eta \alpha} \tau \omega v$ M $\alpha \theta \eta \mu \alpha \tau 1 \kappa \omega ́ v$.

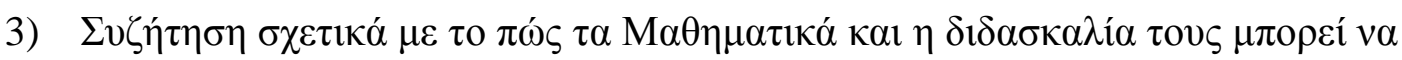

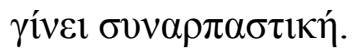

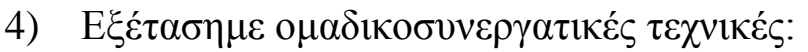

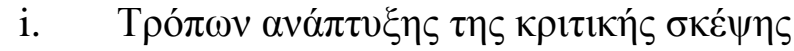

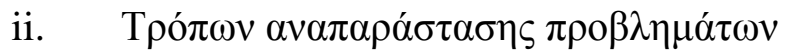

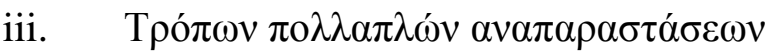

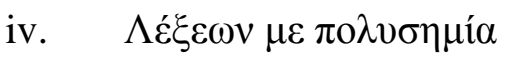

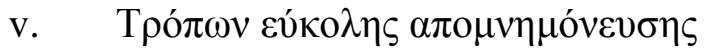

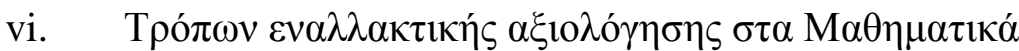

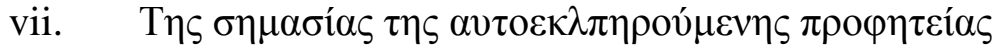

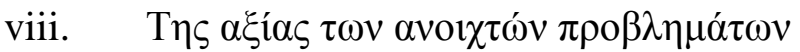

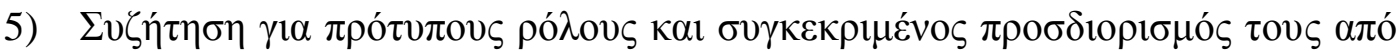

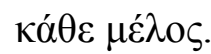

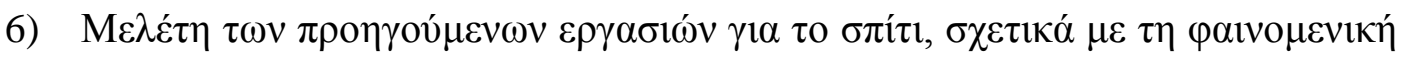
$\delta v \sigma \kappa о \lambda i ́ \alpha \sigma \tau \alpha \mathrm{M} \alpha \theta \eta \mu \alpha \tau \iota \kappa \alpha ́$ 


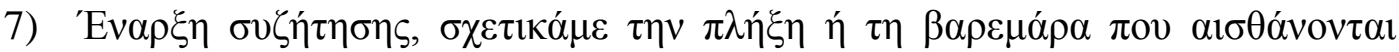

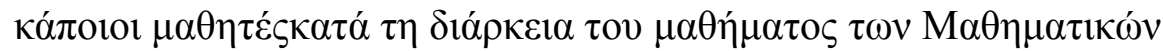

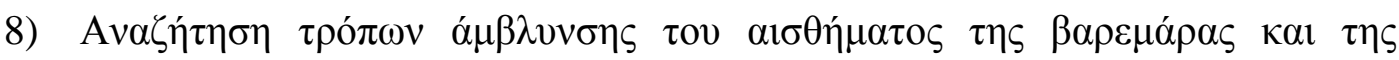

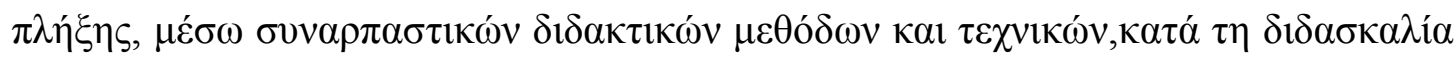
$\tau \omega v \mathrm{M} \alpha \theta \eta \mu \alpha \tau \iota \kappa \omega ́ v$.

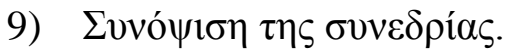

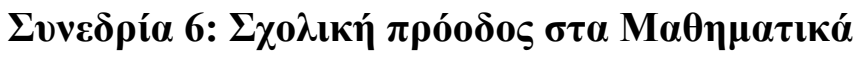

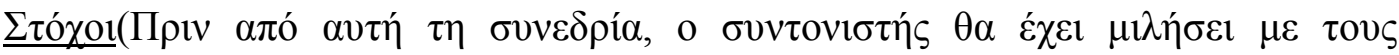

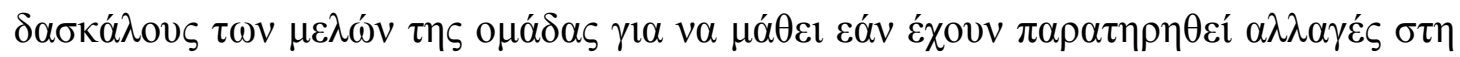

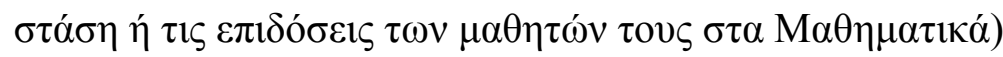

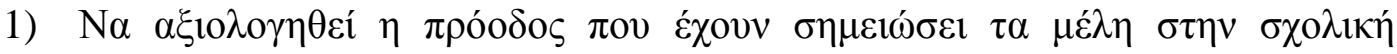

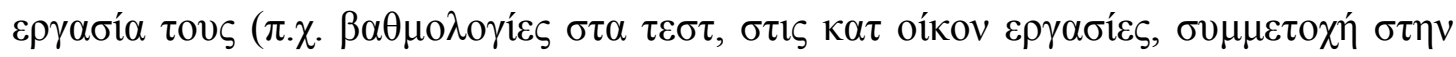

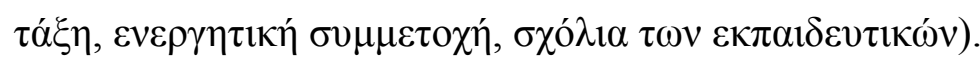

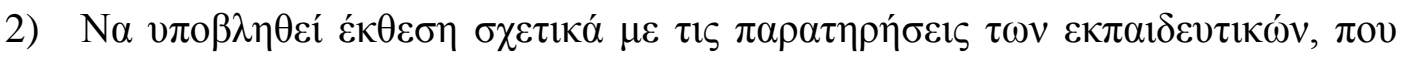

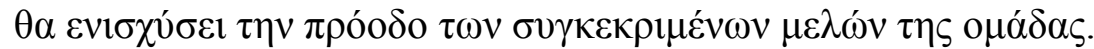

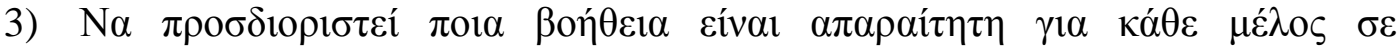

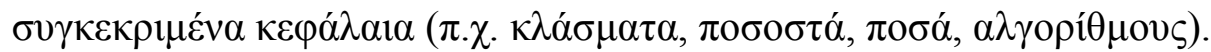

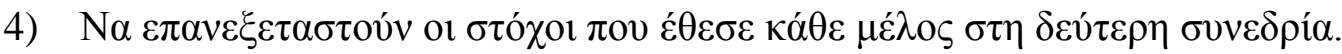

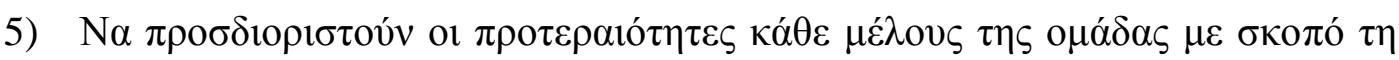

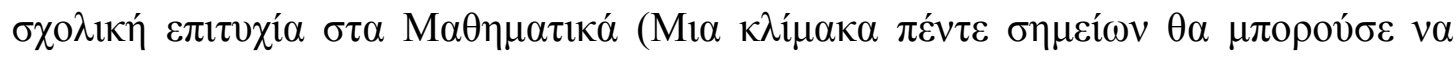

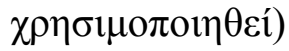

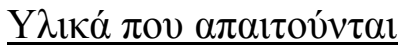

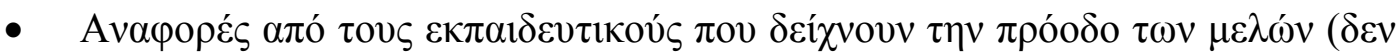

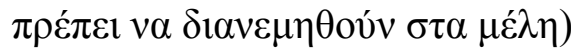

\section{$\underline{\Sigma v \gamma \kappa \varepsilon \kappa \rho \iota \mu \varepsilon ́ v \varepsilon \varsigma \zeta ~ \sigma \tau \rho \alpha \tau \eta \gamma \iota \kappa \varepsilon ́ \varsigma}$}

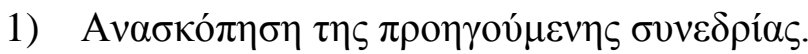

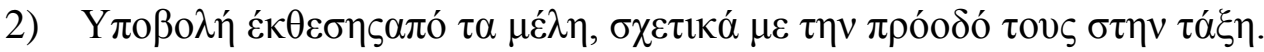

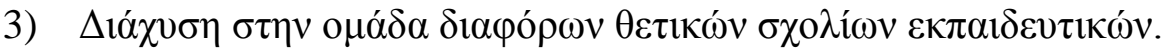




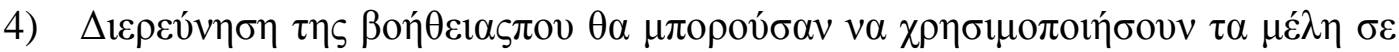

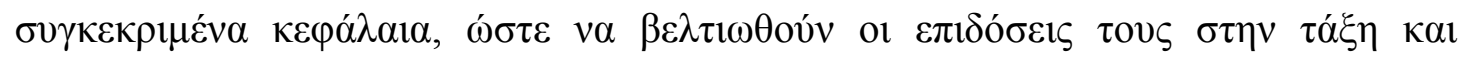

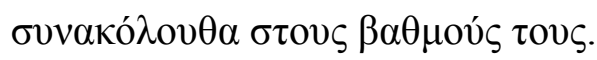

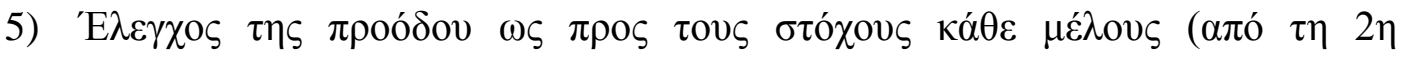
$\sigma v v \varepsilon \delta \rho i ́ \alpha)$.

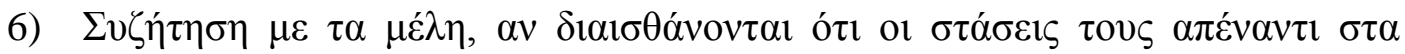

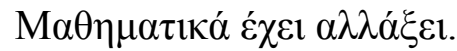

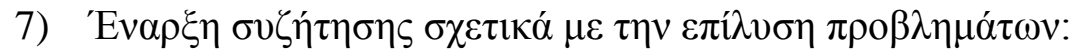

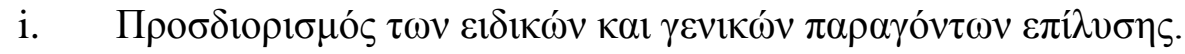

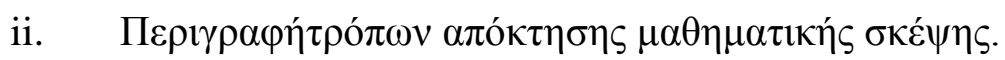

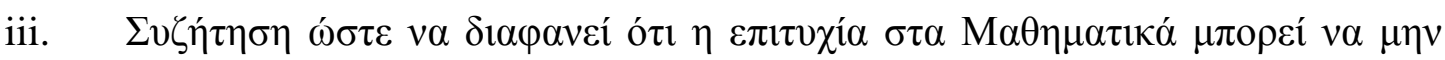

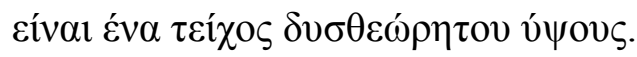

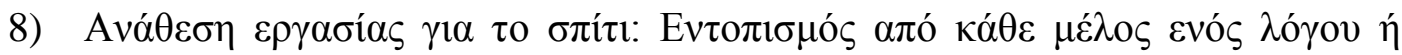

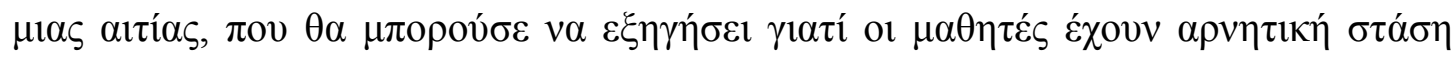

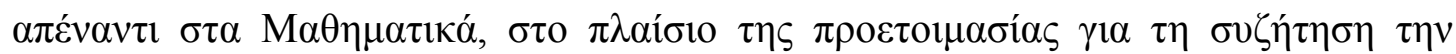
$\varepsilon \pi$ $\mu \varepsilon v \eta \varepsilon \beta \delta о \mu \alpha ́ \delta \alpha$.

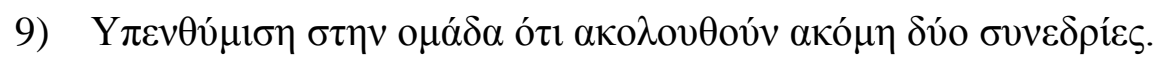

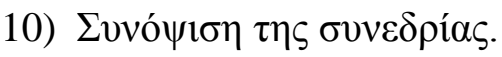

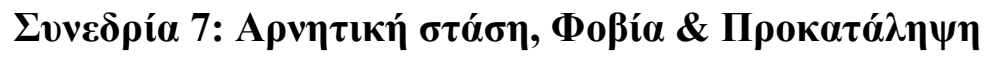

\section{$\underline{\Sigma \tau o ́} \chi 01$}

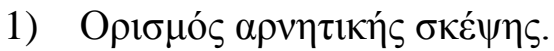

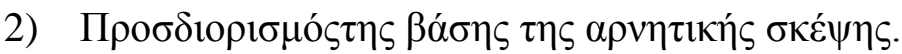

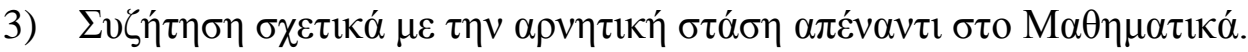

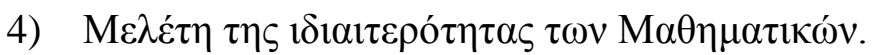

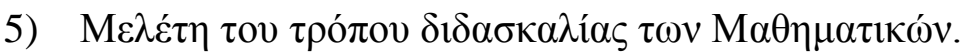

\section{$\underline{\Sigma v \gamma \kappa \varepsilon \kappa \rho \iota \mu \varepsilon ́ v \varepsilon \varsigma \sigma \tau \rho \alpha \tau \eta \gamma \iota \kappa \varepsilon ́ \varsigma}$}

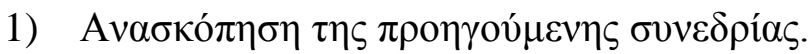

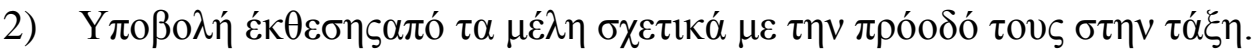

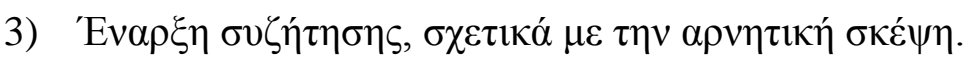




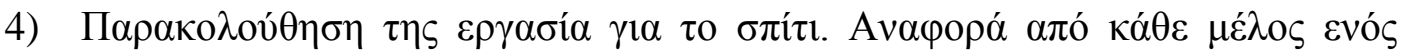

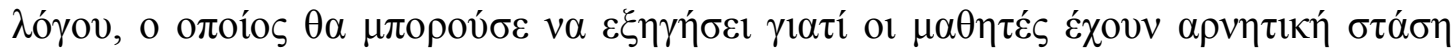

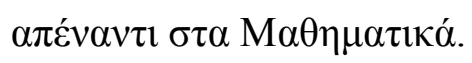

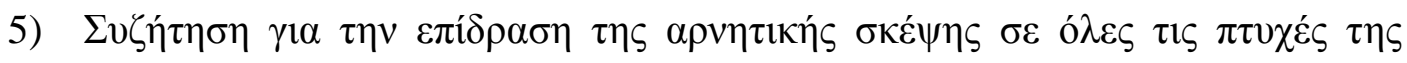
(

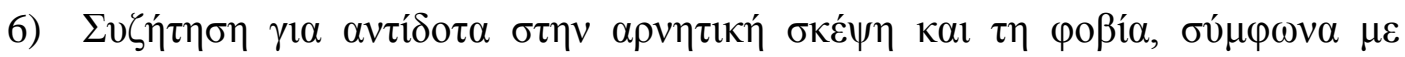

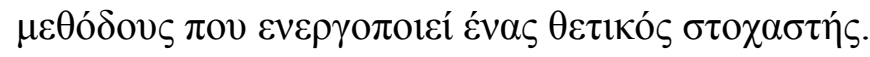

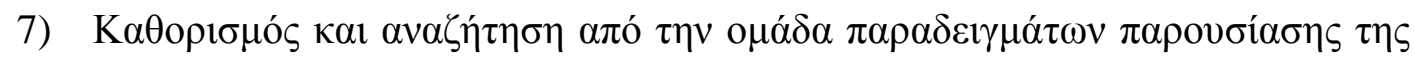

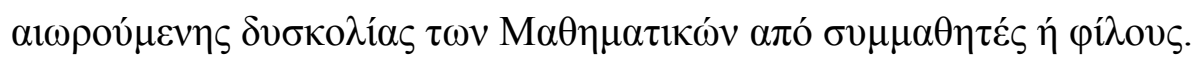

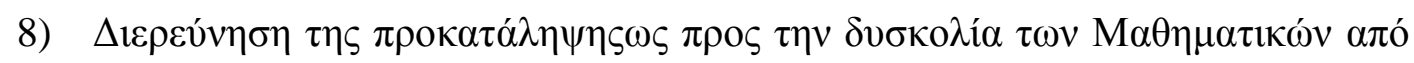

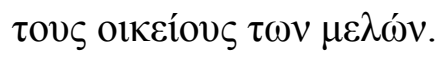

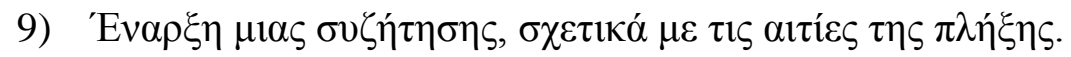

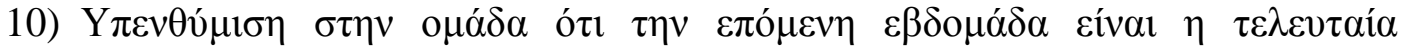

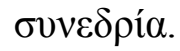

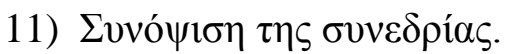

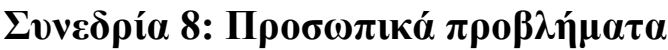

\section{$\underline{\Sigma \tau o ́} \chi 01$}

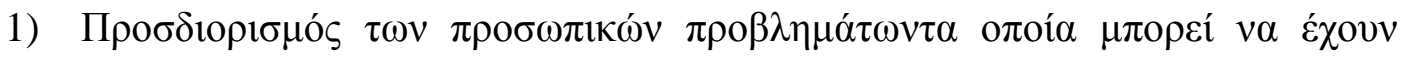

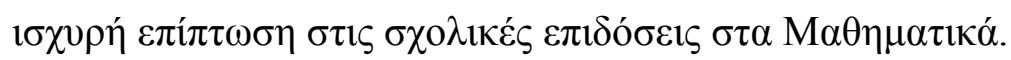

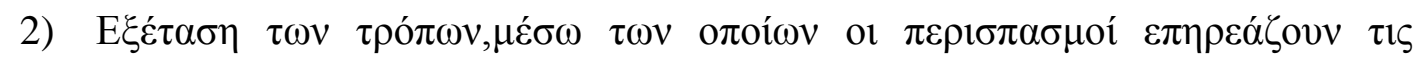
$\sigma \chi 0 \lambda 1 \kappa \varepsilon ́ \varsigma \varepsilon \pi 1 \delta o ́ \sigma \varepsilon 1 \zeta$.

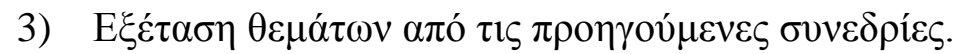

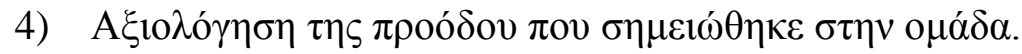

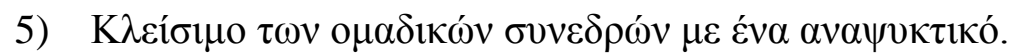

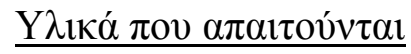

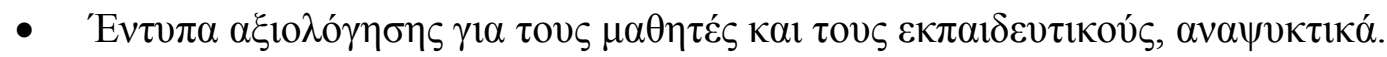

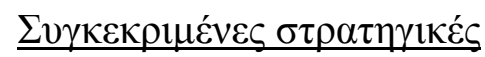

1) Аข

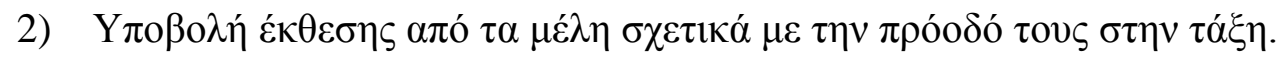




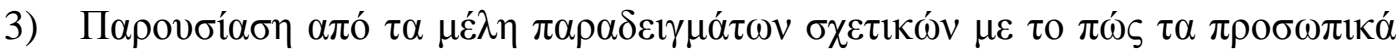

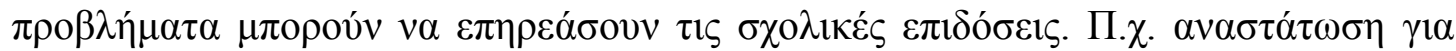

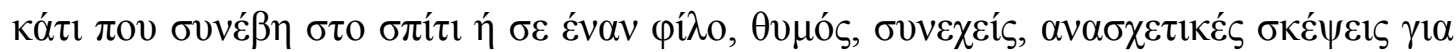

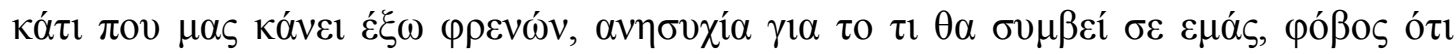

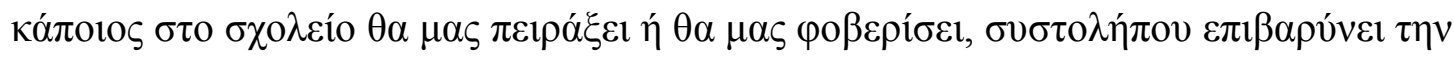

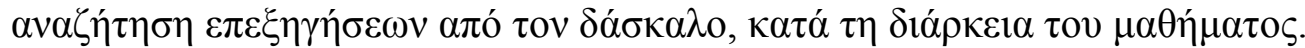

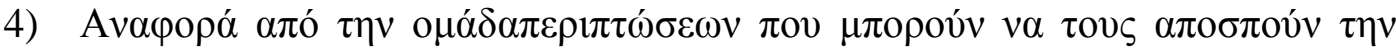

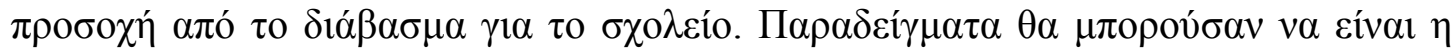

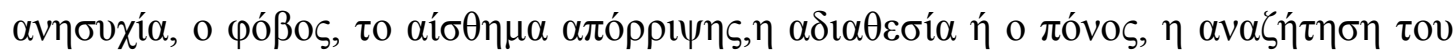

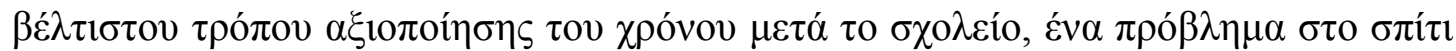
$\kappa . \lambda \pi$.

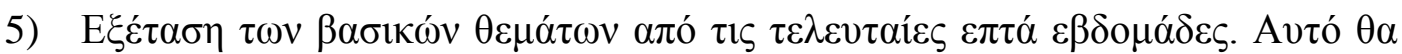

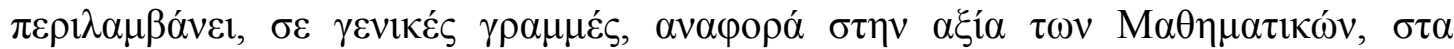

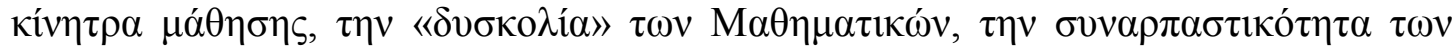

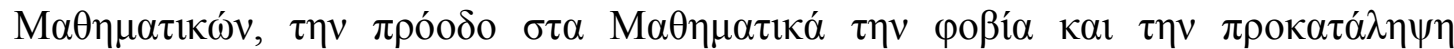

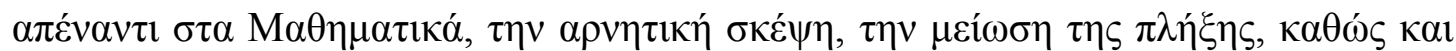

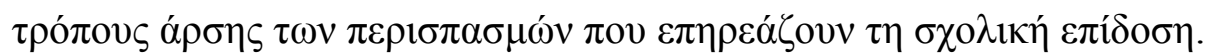

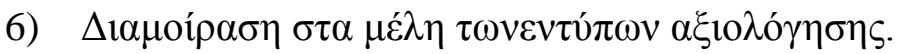

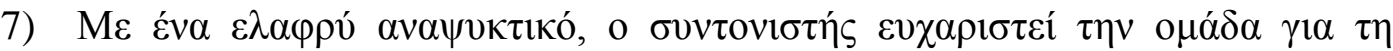

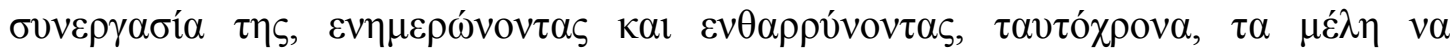

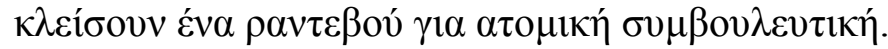

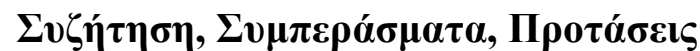

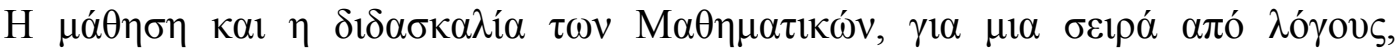

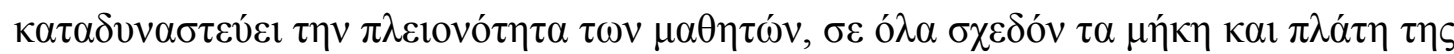

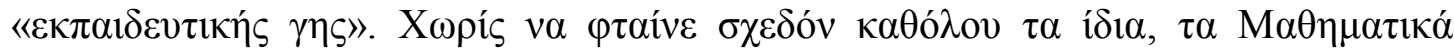

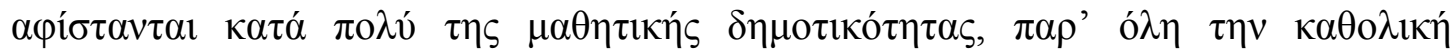

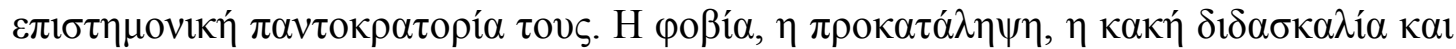

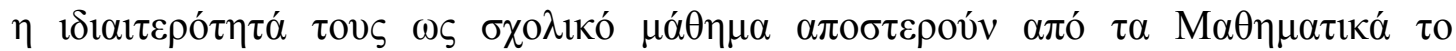

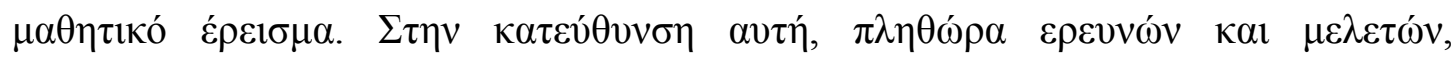

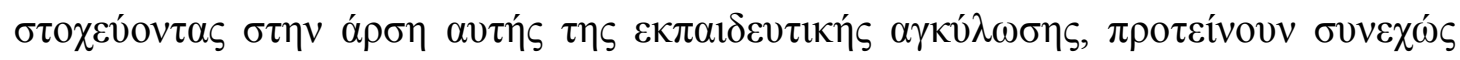

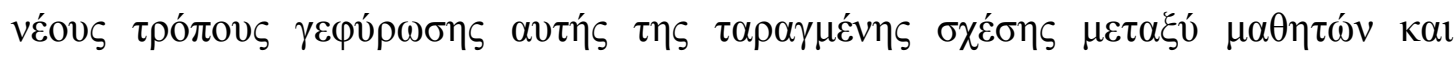

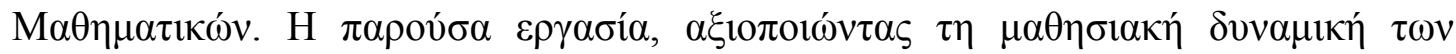




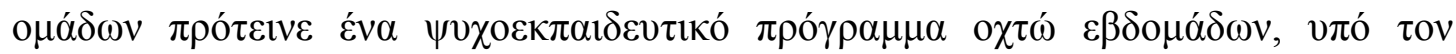

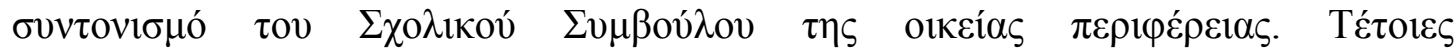

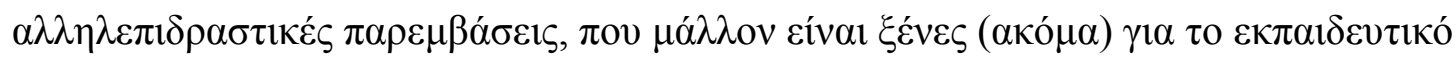

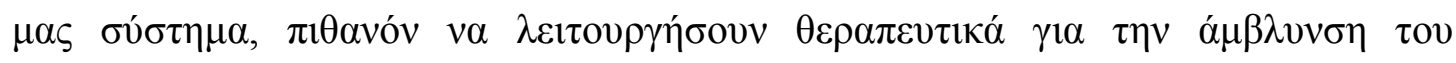

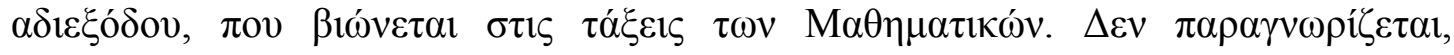

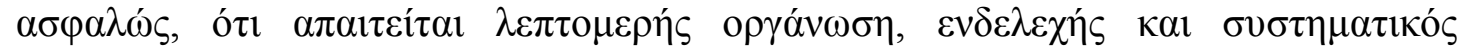

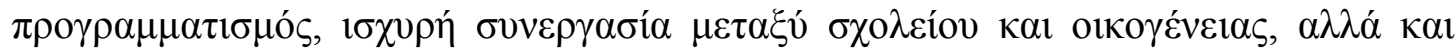

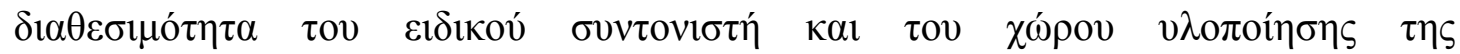

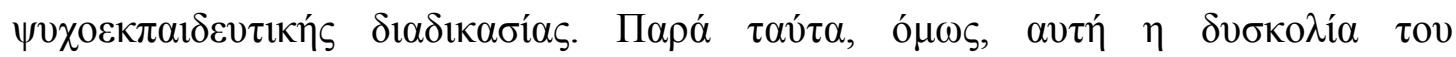

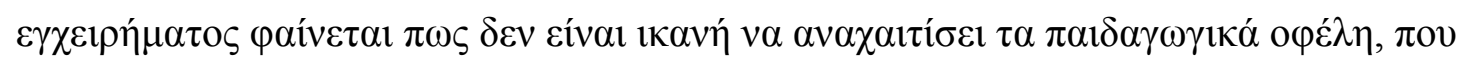

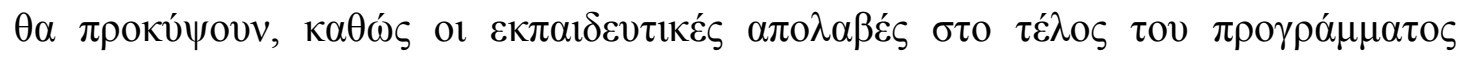

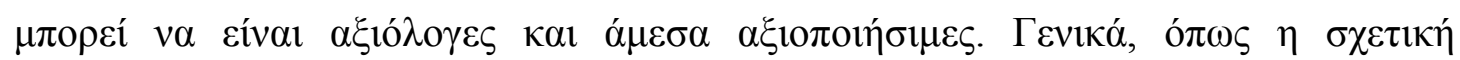

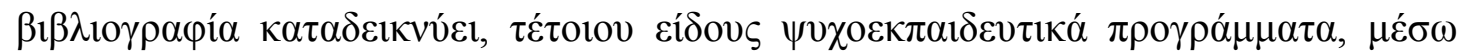

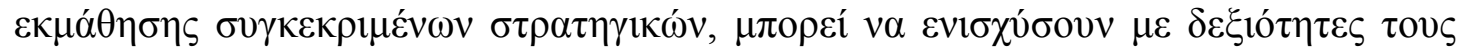

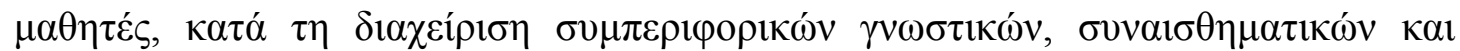

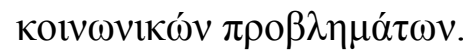

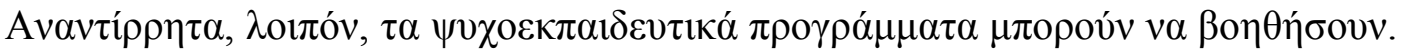

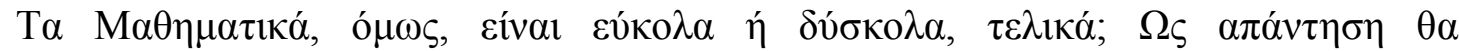

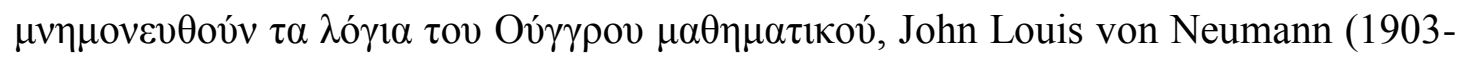

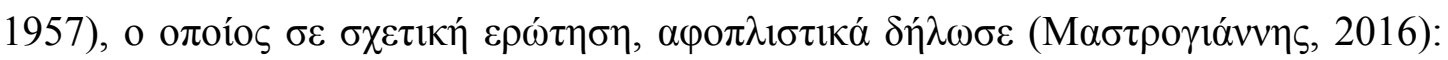

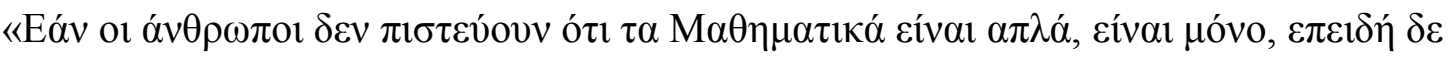

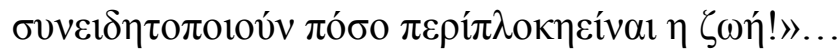

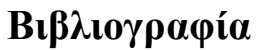

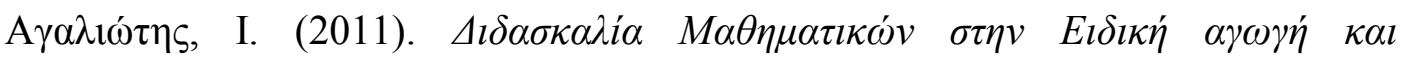

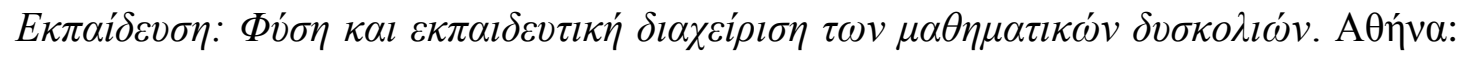

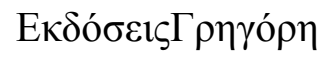

Anderson, A. J. (2001). Psychoeducation: group therapy for the dually diagnosed. International Journal of Psychosocial Rehabilitation, 5, pp. 77-78 
Awasthi, D., Imam, A. \&Singh, G. P. (2015). Relationship between Mathematics Anxiety among Secondary School Students with School Type and Parental Education. Indian Journal of Applied Research, 5(12), pp. 250-253

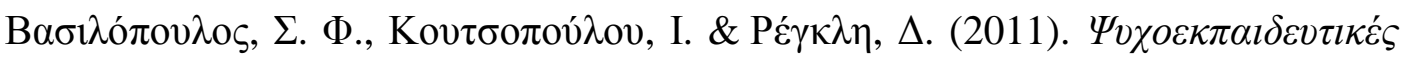

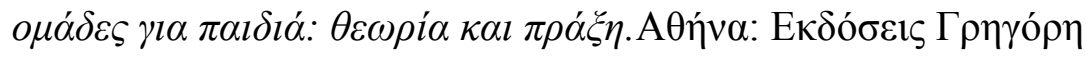

Bore, S. K., Hendricks, L. \&Womack, A. (2013). Psycho-Educational Groups in Schools: The Intervention of Choice. National Forum Journal of Counseling and Addiction, 2(1)

Brown, W. N. (2011). Psychoeducational Groups: Process and Practice. ThirdEdition. Routledge. Taylor\&FrancisGroup

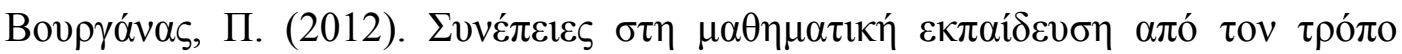

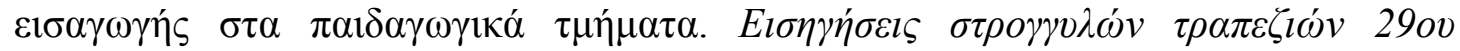

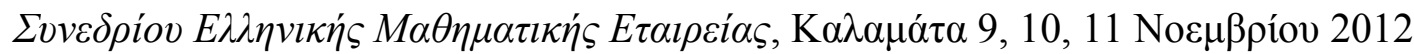

Caruso, H. M. \& Wooley, A. W. (2008). Harnessing the power of emergent interdependence to promote diverse team collaboration. Diversity and Groups, 11, pp. 245-266

Cerrito, P. (1996). Mathematics Across the Curriculum. CollegeTeaching. 44(2)

Champe, J. \& Rubel, D. J. (2012). Application of Focal Conflict Theory to Psychoeducational Groups: Implications for Process, Content and Leadership. The Journal for Specialists in Group Work, 37(1)

Conyne, R. K. (2000). Understanding and Using Group Work. $\Delta$ 1 $\alpha \theta \varepsilon \dot{\sigma} \mu$ o $\alpha \pi$ ó:

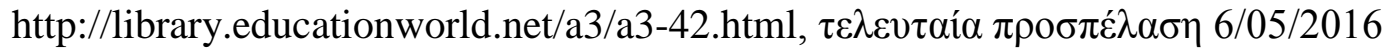

Curtis,W. (2014). How to improve your Math Grades. Berkeley: Occam Press

Delucia-Waack, J. L. (1998). Grouping our groups: What works? The Journal for Specialists in Group Work, 23(2)

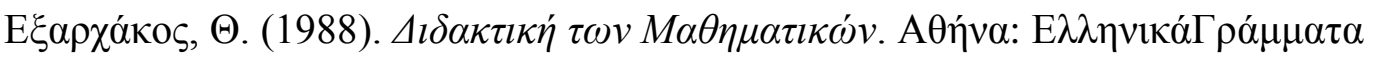


Gerrity, D. A. \& DeLucia-Waack, J. L. (2006) Effectiveness of Groups in the Schools. The Journal for Specialists in Group Work, 32(1), pp. 97-106

Geroski, A. M. \& Kraus, K. L. (2002). Process and Content in School Psychoeducational Groups. The Journal for Specialists in Group Work, 27(2), pp. $233-245$

Greenberg, R. K. (2003). Group counseling in K-12 schools: A handbook for school counselors. Boston, MA: Allyn \& Bacon

Hale, S. \& Cowls, J. (2015). Psychoeducational Groups. InSöderback, I. (Ed), International Handbook of Occupational Therapy Interventions. Second Edition (pp. 509-518). Springer

Hoskonen, K. (2007). Mathematics is - favourite subject, boring or compulsory. In Proceedings of the Fifth Congress of the European Society for Research in Mathematics Education, Larnaca, Cyprus 22 - 26 February, 2007

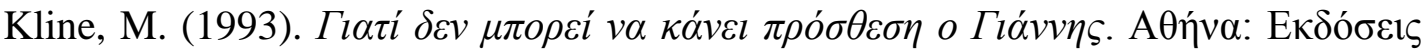
Bávias

Kulm, G. (1999). Evaluating Mathematics Textbooks. Basic Education, 43(9)

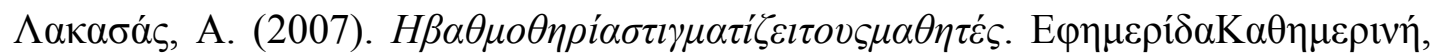
30-12-2007

LaSalvia, T. (1993). Enhancing addiction treatment through Psychoeducational Groups. Journal of Substance Abuse Treatment, 10, pp. 439-444

Legner, P. (2013). The value of teaching mathematics. ( $\Delta \mathrm{t} \alpha \theta \dot{\varepsilon} \sigma 1 \mu \mathrm{oon}$ line: //mathigon.org/resources/valueof-mathematics.pdf, $\pi \rho о \sigma \pi \varepsilon \lambda \dot{\alpha} \sigma \tau \eta \kappa \varepsilon ~ 21 / 5 / 2016$ )

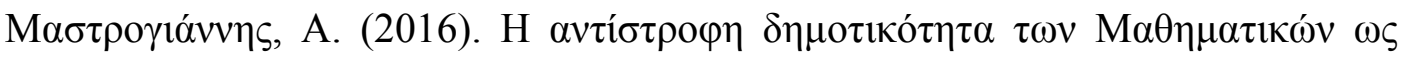

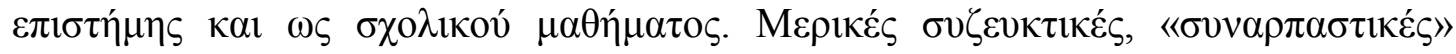

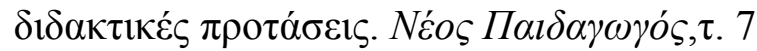




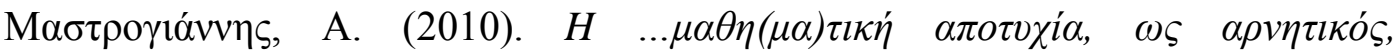

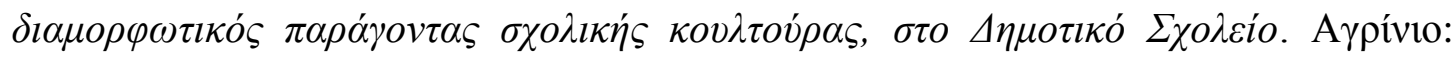

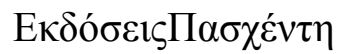

Mastrogiannis, A. \& Trypa, A. (2010). Lateral transfer of learning from software of aesthetic and creative expression to mathematics. In Proceedings of EDULEARN10, International Conference on Education and New Learning Technologies, (pp. 3440-3449), July 5, 6 and 7, 2010, Barcelona, Spain

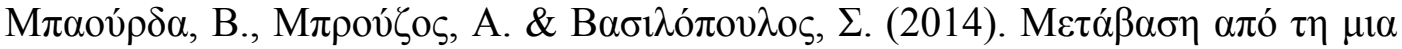

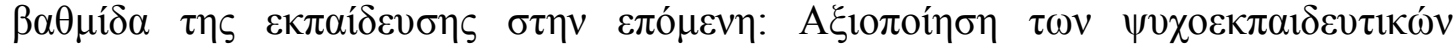

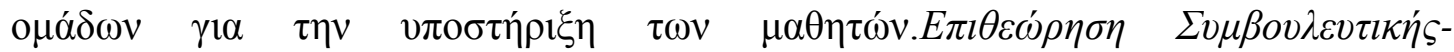

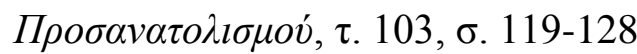

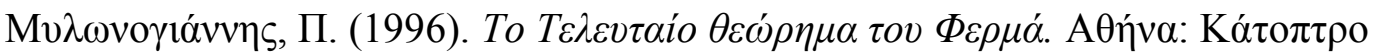

Pantziara, M. \&Philippou, G. (2015). Students' Motivation in the Mathematics Classroom. Revealing Causes and Consequences. International Journal of Science and Mathematics Education, 13(2), pp. 385-411

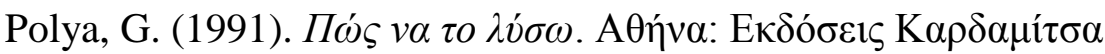

Puteh, M. \&Khalin, S. Z. (2016). Mathematics Anxiety and Its Relationship with the Achievement of Secondary Students in Malaysia. International Journal of Social Science and Humanity, 6(2), pp. 119-122

Russel, H. (1984). Implications of Some Results of the Second International Mathematics Study. In Damerow, P., Dunkley, M., Nebres, B. \& Werry B. (Eds), Report and papers presented in theme group I, «Mathematics for All» at the 5th International Congress on Mathematical Education, Adelaide, August 24-29, 1984, UNESCO

Tang, K. C. (2005). Secondary School Mathematics Curricula. In Bray, M. \& Koo, R. (2004) (Eds), Education and Society in Hong Kong and Macau: Comparative Perspectives on Continuity and Change. Comparative Education Research Centre, Univ. of Hong Kong 
Thompson, P. W., Artigue, M., Törner, G. \& de Shalit, E. (2014). Collaboration between mathematics and mathematics education. In M. Fried \& T. Dreyfus (Eds), Mathematics and mathematics education: Searching for the common ground (pp. 313-333). Berlin: Springer

Tinto, V. (1994). Leaving college: Rethinking the causes and cures of student attrition. Second Edition. Chicago: University of Chicago Press

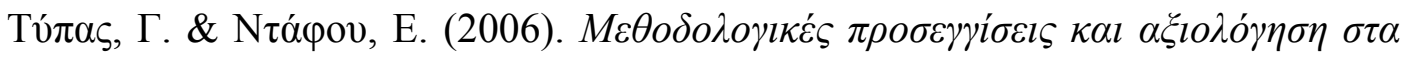

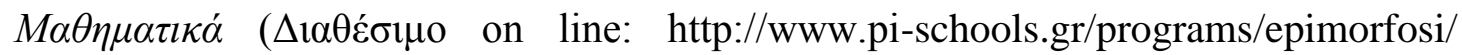

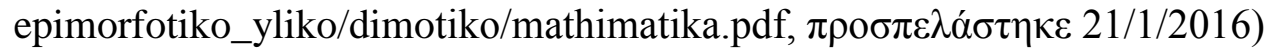

Van Steenbrugge, H., Valcke, M. \& Desoete, A. (2010). Mathematics learning difficulties in primary education: Teachers' professional knowledge and the use of commercially available learning packages. Educational studies, 36, pp. 59-71

Wigfield, A. \& Meece, L. J. (1988). Math Anxiety in Elementary and Secondary School Students. Journal of Educational Psychology, 80(2), pp. 210-216

Yalçın, S., Yavuz, H. C. \& Dibek, I. M. (2015). Content Analysis of Papers Published in Educational Journals with High Impact Factors. Education and Science, 40(182), pp. 1-28

Yalom, I. D. \& Leszcz, M. (2005). The theory and practice of group psychotherapy. FifthEdition. NewYork: BasicBooks 